\title{
Nonmonotonic reasoning from conditional knowledge bases with system W
}

\author{
Christian Komo $^{1} \cdot$ Christoph Beierle $^{1}$
}

Accepted: 27 September 2021 / Published online: 14 December 2021

(C) The Author(s) 2021

\begin{abstract}
For nonmonotonic reasoning in the context of a knowledge base $\mathcal{R}$ containing conditionals of the form If $A$ then usually $B$, system $P$ provides generally accepted axioms. Inference solely based on system $P$, however, is inherently skeptical because it coincides with reasoning that takes all ranking models of $\mathcal{R}$ into account. System $\mathrm{Z}$ uses only the unique minimal ranking model of $\mathcal{R}$, and c-inference, realized via a complex constraint satisfaction problem, takes all c-representations of $\mathcal{R}$ into account. C-representations constitute the subset of all ranking models of $\mathcal{R}$ that are obtained by assigning non-negative integer impacts to each conditional in $\mathcal{R}$ and summing up, for every world, the impacts of all conditionals falsified by that world. While system $\mathrm{Z}$ and c-inference license in general different sets of desirable entailments, the first major objective of this article is to present system $W$. System $\mathrm{W}$ fully captures and strictly extends both system $\mathrm{Z}$ and c-inference. Moreover, system W can be represented by a single strict partial order on the worlds over the signature of $\mathcal{R}$. We show that system $\mathrm{W}$ exhibits further inference properties worthwhile for nonmonotonic reasoning, like satisfying the axioms of system $\mathrm{P}$, respecting conditional indifference, and avoiding the drowning problem. The other main goal of this article is to provide results on our investigations, underlying the development of system $\mathrm{W}$, of upper and lower bounds that can be used to restrict the set of c-representations that have to be taken into account for realizing c-inference. We show that the upper bound of $n-1$ is sufficient for capturing cinference with respect to $\mathcal{R}$ having $n$ conditionals if there is at least one world verifying all conditionals in $\mathcal{R}$. In contrast to the previous conjecture that the number of conditionals in $\mathcal{R}$ is always sufficient, we prove that there are knowledge bases requiring an upper bound of $2^{n-1}$, implying that there is no polynomial upper bound of the impacts assigned to the conditionals in $\mathcal{R}$ for fully capturing c-inference.
\end{abstract}

Keywords Conditional $\cdot$ Conditional knowledge base $\cdot$ Nonmonotonic inference $\cdot$ System $\mathrm{P} \cdot$ System Z · C-representation · C-inference · Constraint satisfaction problem · System W

Christian Komo

christian.komo@web.de

Christoph Beierle

christoph.beierle@fernuni-hagen.de

1 Faculty of Mathematics and Computer Science, FernUniversität in Hagen, 58084 Hagen, Germany 
Mathematics Subject Classification (2010) 68T27 • 68T30 • 68T37

\section{Introduction}

In the area of knowledge representation and reasoning, conditionals play a prominent role. Nonmonotonic reasoning investigates qualitative conditionals of the form "If $A$ then usually $B$ ". Rules of this kind clearly go beyond classical logic because any propositional interpretation either verifies the conditional if both $A$ and $B$ hold in that interpretation, or it falsifies the conditional if $A$ holds and $B$ does not hold, or the conditional is not applicable if $A$ does not hold. Various semantical approaches have been proposed for inductive inferences based on sets of such conditionals. Calculi to compute inductive inferences like Adam's system P [1], probabilistic approaches like p-entailment [16], or possibilistic inference methods [15] have been developed. Further semantical investigations and frameworks include [7, 12, 14, 26, 29-31] as well as inductive methods based on Spohn's ordinal conditional functions (OCFs) [35, 36] like Pearl's system Z [17, 33] or c-representations [19, 20]. OCFs assign a degree of surprise to each world $\omega$ inducing a nonmonotonic inference relation [15, 33, 35]. C-representations are special ranking functions being based on the principle of conditional indifference and exhibiting desirable inference properties [19, 20, 37]. C-inference which was introduced in [2] is the skeptical inference relation taking all c-representations into account.

For the evaluation of nonmonotonic inference relations, many postulates have been proposed. One of the most common sets of postulates are the axioms of system P [1, 26]; in [18], Hawthorne and Makinson call these axioms the "industry standard" for qualitative nonmonotonic inference relations. When comparing two inference relations, we may observe that one relation strictly extends the other one by licensing strictly more inferences, or the two inference relations may be incomparable with respect to set inclusion. For instance, it is known that system $\mathrm{Z}$ and c-inference both satisfy system $\mathrm{P}$ and that in general, they both allow for more entailments from a given set of conditionals than applying the rules of system $\mathrm{P}$ only $[4,17]$. Furthermore, there are system $\mathrm{Z}$ inferences that are not obtained by c-inference, and on the other hand, there are c-inferences that are not system $\mathrm{Z}$ inferences [4].

Because in general, the inferences licensed by system $Z$ and by c-inference both provide sets of desirable entailments that are, however, not identical and that do not have a subset relation between them, the major goal of this article is to develop an inference method, which we call system $W$, fully capturing both system $\mathrm{Z}$ and c-inference. In order to give a first example of an entailment that is missing in one of the two individual approaches, namely in system $\mathrm{Z}$, let us consider the drowning problem [11,33]. An inference relation suffers from the drowning problem if it does not allow to infer properties of a superclass for a subclass that is exceptional with respect to another property because the respective conditional is "drowned" by others. For instance, assume that we have the conditional knowledge that birds usually fly, that penguins usually do not fly, that penguins are usually birds, that birds usually have wings, and that flying things are usually airborn. Thus, penguins are exceptional birds with respect to flying, but not with respect to having wings. So we would reasonably expect that penguins have wings. However, given these five conditionals, system $\mathrm{Z}$ fails to infer that penguins usually have wings. In contrast to system $\mathrm{Z}$ inference, we will show that inference based on system $\mathrm{W}$ does not suffer from the drowning problem.

Besides providing in general different sets of entailments, another notable difference between system $\mathrm{Z}$ and c-inference is that system $\mathrm{Z}$ inference is based on a single unique 
model [33] that can be computed much easier than c-inference which involves many models obtained from the solutions of a complex constraint satisfaction problem (CSP) [4].

Given a consistent, finite set of conditionals $\mathcal{R}$, called a knowledge base, there are infinitely many c-representations for $\mathcal{R}$. These c-representations for $\mathcal{R}$ can be obtained from the solutions of a constraint satisfaction problem $C R(\mathcal{R})$ over the natural numbers; $C R(\mathcal{R})$ can also be employed for realizing c-inference [4]. The solutions of $C R(\mathcal{R})$ yield so-called impacts that are non-negative integers assigned to each of the conditionals in $\mathcal{R}$; the c-representation induced by these impacts is then obtained by summing up all impacts of those conditionals in $\mathcal{R}$ which are falsified by the given world.

By introducing a natural number $u$ as an upper bound for the impacts being solutions of $C R(\mathcal{R})$ and thus by restricting the set of solutions, a finite domains constraint satisfaction problem $C R^{u}(\mathcal{R})$ is obtained. In [4], c-inference under a maximal impact factor $u$ is introduced as skeptical inference taking not all c-representations, but only those crepresentations which are solutions of the restricted constraint satisfaction problem $C R^{u}(\mathcal{R})$ into account. Besides introducing system $\mathrm{W}$, the second major objective of this article is to investigate upper and lower bounds $u \in \mathbb{N}$ that are sufficient for a knowledge base $\mathcal{R}$ in the sense that c-inference under the maximal impact $u$ fully realizes skeptical c-inference [8].

In summary, this article provides the following main contributions:

- We formulate and prove a criterion such that $u=n-1$ is sufficient for a knowledge base $\mathcal{R}$ having $n$ conditionals: If there is a world verifying all conditionals in $\mathcal{R}$.

- While all experiments made with the reasoning platform InfOCF in [6, 9] supported the conjecture that a maximal impact $u=n$ is sufficient for $\mathcal{R}$ if $\mathcal{R}$ has $n$ conditionals, for every $n \in \mathbb{N}$, here we construct a knowledge base with $n$ conditionals such that $2^{n-1}$ is minimally sufficient. Consequently, there is no polynomial upper bound for $u \in \mathbb{N}$ to be sufficient for all knowledge bases with $n$ conditionals.

- We introduce the preferred structure relation $<_{\mathcal{R}}^{\mathrm{w}}$ on worlds based on the notions of tolerance, underlying system $\mathrm{Z}$, and on the verification/falsification behavior of a knowledge base $\mathcal{R}$ that is crucial for c-representations.

- By employing $<_{\mathcal{R}}^{\mathrm{W}}$, we develop a new inference relation, called system $W$, which does not require, in contrast to skeptical c-inference, to solve a complex constraint satisfaction problem, but relies, similar to system $\mathrm{Z}$, on just one single ordering relation of the worlds.

- We prove that system $\mathrm{W}$ inference captures and strictly extends both system $\mathrm{Z}$ inference and also skeptical c-inference.

- We show that system $\mathrm{W}$ exhibits further desirable properties, like satisfying the axioms of system $\mathrm{P}$, respecting conditional indifference, and avoiding the bdrowning problem.

This article is a revised and largely extended version of [25]. The extensions include in particular the development of a refined constraint satisfaction problem associated to $\mathcal{R}$ and establishing its exact relation to $C R(\mathcal{R})$, and the preferred structure relation and system $\mathrm{W}$. Furthermore, this article also revises and extends our work on system W in [24], in particular by providing a full formal proof that system $\mathrm{W}$ inference captures skeptical c-inference and satisfies desirable properties established for nonmonotonic reasoning.

The rest of the paper is organized as follows. After briefly recalling the basics of conditional logic, ranking functions, and system $\mathrm{Z}$ in Section 2, we present c-representations and their characterization as a $\operatorname{CSP} C R(\mathcal{R})$ as well as c-inference in Section 3. We deal with resource bounded c-inference and the concept of sufficient and regular bounds for finite domain CSPs in Section 4. In Section 5, we present a formula to obtain solutions to $C R(\mathcal{R})$. The topic of Section 6 is a criterion on a knowledge base $\mathcal{R}$ with $n$ conditionals such that $n-1$ is sufficient for $\mathcal{R}$. In Section 7, we deal with the construction of knowledge 
bases whose existence will establish the exponential lower bound for skeptical c-inference under maximal impact $u$ to be equivalent to skeptical c-inference for all knowledge bases. Section 8 defines the preferred structure on worlds and elaborates characteristics of it that are exloited for the design of system W. In Section 9, we introduce system W and discuss and prove its main properties. In Section 10, we conclude and point out future work.

\section{Conditional logic and OCFs}

Let $\Sigma=\left\{v_{1}, \ldots, v_{m}\right\}$ be a propositional alphabet. A literal is the positive $\left(v_{i}\right)$ or negated $\left(\neg v_{i}\right)$ form of a propositional variable, $\dot{v}_{i}$ stands for either $v_{i}$ or $\neg v_{i}$. From these we obtain the propositional language $\mathcal{L}$ as the set of formulas of $\Sigma$ closed under negation $\neg$, conjunction $\wedge$, disjunction $\vee$, and material implication $\Rightarrow$. For shorter formulas, we abbreviate conjunction by juxtaposition (i.e., $A B$ stands for $A \wedge B$ ), and negation by overlining (i.e., $\bar{A}$ is equivalent to $\neg A$ ). Let $\Omega_{\Sigma}$ denote the set of possible worlds over $\mathcal{L} ; \Omega_{\Sigma}$ will be taken here simply as the set of all propositional interpretations over $\mathcal{L}$ and can be identified with the set of all complete conjunctions over $\Sigma$; we will often just write $\Omega$ instead of $\Omega_{\Sigma}$. For $\omega \in \Omega, \omega \models A$ means that the propositional formula $A \in \mathcal{L}$ holds in the possible world $\omega$. With $\Omega_{A}=\left\{\omega \in \Omega_{\Sigma} \mid \omega \models A\right\}$, we denote the set of all worlds in which $A$ holds.

A conditional $(B \mid A)$ with $A, B \in \mathcal{L}$ encodes the defeasible rule "if $A$ then normally $B$ " and is a trivalent logical entity with the evaluation $[13,19]$

$$
\llbracket(B \mid A) \rrbracket_{\omega}=\left\{\begin{array}{llll}
v & \text { iff } & \omega \models A B & \text { (verification), } \\
\mathrm{f} & \text { iff } & \omega \models A & \text { (falsification), } \\
- & \text { iff } & \omega \models \bar{A} & \text { (not applicable). }
\end{array}\right.
$$

An ordinal conditional function (OCF, ranking function) [35, 36] is a function $\kappa: \Omega \rightarrow$ $\mathbb{N}_{0} \cup\{\infty\}$ that assigns to each world $\omega \in \Omega$ an implausibility rank $\kappa(\omega)$ : the higher $\kappa(\omega)$, the more surprising $\omega$ is. OCFs have to satisfy the normalization condition that there has to be a world that is maximally plausible, i.e., $\kappa^{-1}(0) \neq \emptyset$. The rank of a formula $A$ is defined by $\kappa(A)=\min \{\kappa(\omega) \mid \omega \models A\}$. An OCF $\kappa$ accepts a conditional $(B \mid A)$, denoted by $\kappa \models(B \mid A)$, if the verification of the conditional is less surprising than its falsification, i.e.,

$$
\kappa \models(B \mid A) \quad \text { iff } \quad \kappa(A B)<\kappa(A \bar{B}) .
$$

This can also be understood as a nonmonotonic inference relation between the premise $A$ and the conclusion $B$ : Basically, we say that $A \kappa$-entails $B$, written $A \sim^{\kappa} B$, if $\kappa$ accepts the conditional $(B \mid A)$; formally this if given by

$$
A \sim{ }^{\kappa} B \text { iff } A \equiv \perp \text { or } \kappa(A B)<\kappa(A \bar{B}) .
$$

Note that the reason for including the disjunctive condition in (3) is to ensure that $\sim^{\kappa}$ satisfies supraclassicality, i.e., $A \models B$ implies $A \sim^{\kappa} B$, also for the case $A \equiv \perp$ as it is required, for instance, by the reflexivity axiom $A \sim A$ of system $\mathrm{P}[1,26]$. For nonmonotonic reasoning with qualitative conditionals as given above, the postulates of system $\mathrm{P}$ (Fig. 1) are generally regarded as a core any nonmonotonic system should satisfy; in [18], Hawthorne and Makinson call these axioms the "industry standard" for qualitative nonmonotonic inference.

Let us remark that $\kappa$-entailment is based on the total preorder on possible worlds induced by a ranking function and can be expressed equivalently by:

$$
A \sim^{\kappa} B \text { iff } \forall \omega^{\prime} \in \Omega_{A \bar{B}} \exists \omega \in \Omega_{A B} \kappa(\omega)<\kappa\left(\omega^{\prime}\right) .
$$




$\begin{array}{ll}\text { Reflexivity } & : A \sim A \\ \text { Cut } & : \quad \frac{A \wedge B \sim C, A \sim B}{A \sim C} \\ \text { Cautious Monotony } & : \quad \frac{A \sim B, A \sim C}{A \wedge B \sim C} \\ \text { Right Weakening } & : \frac{A \sim B, B \models C}{A \sim C} \\ \text { Left Logical Equivalence } & : \frac{\models A \equiv B, A \sim C}{B \sim C} \\ \text { Or } & : \quad \frac{A \sim C, B \sim C}{A \vee B \sim C}\end{array}$

Fig. 1 Axioms of system P [26]

The acceptance relation is extended as usual to a set $\mathcal{R}$ of conditionals, called a knowledge base, by defining $\kappa \models \mathcal{R}$ iff $\kappa \models(B \mid A)$ for all $(B \mid A) \in \mathcal{R}$. This is synonymous to saying that $\kappa$ is admissible with respect to $\mathcal{R}$ [17], or that $\kappa$ is a ranking model of $\mathcal{R}$. $\mathcal{R}$ is consistent iff it has a ranking model.

Two inference relations which are defined by specific OCFs obtained from a knowledge base $\mathcal{R}$ have received some attention: system $Z$ [33] and c-representations [19, 20], or the induced inference relations, respectively, both show excellent inference properties. We recall both approaches briefly.

While there are many ways to define a nonmonotonic inference relation induced by a knowledge base $\mathcal{R}$ two approaches have received particular attention, system $\mathrm{P}$ inference and system $\mathrm{Z}$ inference. We recall both approaches briefly.

For the system $\mathrm{P}$ inference relation based on $\mathcal{R}$, denoted by $\sim_{\mathcal{R}}^{P}$, the inference $A \sim_{\mathcal{R}}^{P} B$ holds if $A \sim{ }_{\mathcal{R}}^{\kappa} B$ holds for all ranking models of $\mathcal{R}$.

On the other hand, system $Z$ [33] is based upon the ranking function $\kappa^{Z}$, which is the unique Pareto-minimal OCF that accepts $\mathcal{R}$. The definition of $\kappa^{Z}$ crucially relies on the notion of tolerance. A conditional $(B \mid A)$ is tolerated by a set of conditionals $\mathcal{R}$ if there is a world $\omega \in \Omega$ such that $\omega \models A B$ and $\omega \models \bigwedge_{i=1}^{n}\left(A_{i} \Rightarrow B_{i}\right)$, i.e., iff $\omega$ verifies $(B \mid A)$ and does not falsify any conditional in $\mathcal{R}$. For every consistent knowledge base, the notion of tolerance yields an ordered partition $\left(\mathcal{R}_{0}, \ldots, \mathcal{R}_{k}\right)$ of $\mathcal{R}$, where each $\mathcal{R}_{i}$ is tolerated by $\bigcup_{j=i}^{k} \mathcal{R}_{j}$. The inclusion-maximal partition of $\mathcal{R}$, in the following denoted by $O P(\mathcal{R})=\left(\mathcal{R}_{0}, \ldots, \mathcal{R}_{k}\right)$, is the ordered partition of $\mathcal{R}$ where each $\mathcal{R}_{i}$ is the (with respect to set inclusion) maximal subset of $\bigcup_{j=i}^{k} \mathcal{R}_{j}$ that is tolerated by $\bigcup_{j=i}^{k} \mathcal{R}_{j}$. This partitioning is unique due to the maximality and can be computed using the consistency test algorithm given in [17]; for an inconsistent knowledge base $\mathcal{R}, O P(\mathcal{R})$ does not exist. Using $O P(\mathcal{R})=\left(\mathcal{R}_{0}, \ldots, \mathcal{R}_{k}\right)$, the system $\mathrm{Z}$ ranking function $\kappa^{Z}$ is defined by

$$
\kappa^{Z}(\omega):= \begin{cases}0, & \text { if } \omega \text { does not falsify any conditional } r \in \mathcal{R}, \\ 1+\max _{\substack{1 \leq i \leq n \\ \omega \models A_{i} \overline{B_{i}}}} Z\left(r_{i}\right), & \text { otherwise, }\end{cases}
$$

where the function $Z: \mathcal{R} \rightarrow \mathbb{N}_{0}$ is given by $Z\left(r_{i}\right)=j$ if $r_{i} \in \mathcal{R}_{j}$. 
Definition 1 (system $Z$ inference, $\sim_{\mathcal{R}}^{Z}[17,33]$ ) Let $\mathcal{R}$ be a knowledge base and let $A$, $B$ be formulas. We say that $B$ is a system $Z$ inference of $A$ in the context of $\mathcal{R}$, denoted by $A \sim{ }_{\mathcal{R}}^{Z} B$, iff $A \sim{ }^{Z} B$ holds.

Example 1 ( $\left.\mathcal{R}_{\text {bird }}\right)$ To illustrate the definitions and concepts presented in this paper let us consider an instance of the well known penguin bird example. This example will be a running example and will be continued throughout the paper. Consider the propositional alphabet $\Sigma=\{p, b, f\}$ representing whether something is a penguin $(p)$, whether it is a bird $(b)$, or whether it can fly $(f)$. The set $\Omega$ is given as follows

$$
\Omega=\{p b f, p b \bar{f}, p \bar{b} f, p \bar{b} \bar{f}, \bar{p} b f, \bar{p} b \bar{f}, \bar{p} \bar{b} f, \bar{p} \bar{b} \bar{f}\} .
$$

The knowledge base $\mathcal{R}_{\text {bird }}=\left\{r_{1}, r_{2}, r_{3}, r_{4}\right\}$ is given by

$$
\begin{array}{cl}
r_{1}=(f \mid b) & \text { "Birds usually fly", } \\
r_{2}=(\bar{f} \mid p) & \text { "Penguins usually do not fly", } \\
r_{3}=(\bar{f} \mid b p) & \text { "Penguins which are also birds usually do not fly", } \\
r_{4}=(b \mid p) & \text { "Penguins are usually birds". }
\end{array}
$$

For $\mathcal{R}_{0}=\{(f \mid b)\}$ and $\mathcal{R}_{1}=\mathcal{R}_{\text {bird }} \backslash \mathcal{R}_{0}$ we have the ordered partitioning $\left(\mathcal{R}_{0}, \mathcal{R}_{1}\right)$ such that every conditional in $\mathcal{R}_{0}$ is tolerated by $\mathcal{R}_{0} \cup \mathcal{R}_{1}=\mathcal{R}_{\text {bird }}$ and every conditional in $\mathcal{R}_{1}$ is tolerated by $\mathcal{R}_{1}$. For instance, $(f \mid b)$ is tolerated by $\mathcal{R}_{\text {bird }}$ since there is, for example, the world $\bar{p} b f$ with $\bar{p} b f \models b f$ as well as $\bar{p} b f \models(p \Rightarrow \bar{f}) \wedge(p b \Rightarrow \bar{f}) \wedge(p \Rightarrow b)$. Furthermore $\left(\mathcal{R}_{0}, \mathcal{R}_{1}\right)$ is indeed the inclusion-maximal partition of $\mathcal{R}$. Because there is an ordered partition for it, $\mathcal{R}_{\text {bird }}$ is consistent.

Table 1 shows the system $\mathrm{Z}$ ranking function $\kappa^{Z}$ for $\mathcal{R}_{\text {bird }}$. For instance, we have $\kappa^{Z}(\bar{p} b \bar{f})=1$ since the world $\omega=\bar{p} b \bar{f}$ falsifies the conditional $r_{1}=(f \mid b) \in \mathcal{R}_{0}$ but does not falsify any conditional from $\mathcal{R}_{1}$. We obtain the system $\mathrm{Z}$ inference $\bar{p} b \sim \stackrel{\mathcal{R}}{\text { bird }}_{\mathrm{Z}} f$, i.e., that birds, which are no penguins, usually fly. Indeed, this follows from

$$
\kappa^{Z}(\bar{p} b f)=0<1=\kappa^{Z}(\bar{p} b \bar{f}) .
$$

In the following section, we will turn to an inference approach that does not not rely on all models nor on any specific single model of $\mathcal{R}$, but on a specific subclass of ranking models of $\mathcal{R}$.

\section{Inference based on c-representations}

Among the OCF models of $\mathcal{R}$, c-representations $[19,20]$ are special ranking models that are governed by the principle of conditional indifference: If two worlds $\omega_{1}$ and $\omega_{2}$ exhibit the same behavior with respect to falsifying the conditionals in a knowledge base $\mathcal{R}$, then a c-representation for $\mathcal{R}$ should treat $\omega_{1}$ and $\omega_{2}$ in the same way and should associate the same rank to both worlds. In order to obtain this behavior, each conditional in $\left(B_{i} \mid A_{i}\right)$ in $\mathcal{R}$ is assigned a penalty value $\eta_{i}$. The rank of a world under a c-representation is then given by

Table 1 System $Z$ ranking model $\kappa^{Z}$ for the knowledge base $\mathcal{R}_{\text {bird }}$ in Example 1

\begin{tabular}{lllllllll}
\hline$\omega$ & $p b f$ & $p b \bar{f}$ & $p \bar{b} f$ & $p \bar{b} \bar{f}$ & $\bar{p} b f$ & $\bar{p} b \bar{f}$ & $\bar{p} \bar{b} f$ & $\bar{p} \bar{b} \bar{f}$ \\
\hline$\kappa^{Z}(\omega)$ & 2 & 1 & 2 & 2 & 0 & 1 & 0 & 0 \\
\hline
\end{tabular}


summing up the penalty values of all conditionals falsified by that world. In this way, it is ensured that $\omega_{1}$ and $\omega_{2}$ get the same rank if they falsify precisely the same conditionals of $\mathcal{R}$. The penalty values are non-negativeintegers and will be called impacts in the following.

Definition 2 (c-representation [19, 20]) A c-representation of a knowledge base $\mathcal{R}$ is a ranking function $\kappa \vec{\eta}$ constructed from $\vec{\eta}=\left(\eta_{1}, \ldots, \eta_{n}\right)$ with integer impacts $\eta_{i} \in \mathbb{N}_{0}, i \in$ $\{1, \ldots, n\}$ assigned to each conditional $\left(B_{i} \mid A_{i}\right)$ such that $\kappa$ accepts $\mathcal{R}$ and is given by:

$$
\kappa_{\vec{\eta}}(\omega)=\sum_{\substack{1 \leq i \leq n \\ \omega \models A_{i} \bar{B}_{i}}} \eta_{i}
$$

We will denote the set of all c-representations of $\mathcal{R}$ by $\mathcal{O}(C R(\mathcal{R}))$.

In [4] a modeling of c-representations as solutions of a constraint satisfaction problem $C R(\mathcal{R})$ is given and shown to be sound and complete with respect to the set of all c-representations of $\mathcal{R}$.

Definition $3(\boldsymbol{C R}(\mathcal{R})[2,4])$ Let $\mathcal{R}=\left\{\left(B_{1} \mid A_{1}\right), \ldots,\left(B_{n} \mid A_{n}\right)\right\}$. The constraint satisfaction problem for c-representations of $\mathcal{R}$, denoted by $C R(\mathcal{R})$, on the constraint variables $\left\{\eta_{1}, \ldots, \eta_{n}\right\}$ ranging over $\mathbb{N}_{0}$ is given by the conjunction of the constraints, for all $i \in$ $\{1, \ldots, n\}$ :

$$
\eta_{i}>\min _{\omega \models A_{i} B_{i}} \sum_{\substack{j \neq i \\ \omega \models A_{j} \overline{B_{j}}}} \eta_{j}-\min _{\omega \models A_{i} \bar{B}_{i}} \sum_{\substack{j \neq i \\ \omega \models A_{j} \overline{B_{j}}}} \eta_{j}
$$

A solution of $C R(\mathcal{R})$ is an $n$-tuple $\left(\eta_{1}, \ldots, \eta_{n}\right) \in \mathbb{N}_{0}^{n}$. For a constraint satisfaction problem $C S P$, the set of solutions is denoted by $\operatorname{Sol}(C S P)$. Thus, with $\operatorname{Sol}(\operatorname{CR}(\mathcal{R}))$ we denote the set of all solutions of $C R(\mathcal{R})$. Let us recall the soundness and completeness of constructing c-representations by integer impacts from solutions of $C R(\mathcal{R})$.

Proposition $1[2,4]$ Let $\mathcal{R}=\left\{\left(B_{1} \mid A_{1}\right), \ldots,\left(B_{n} \mid A_{n}\right)\right\}$ be a knowledge base. Then we have

$$
\mathcal{O}(C R(\mathcal{R}))=\left\{\kappa_{\vec{\eta}} \mid \vec{\eta} \in \operatorname{Sol}(C R(\mathcal{R}))\right\}
$$

where $\kappa \vec{\eta}$ is defined as in (6).

Let us illustrate c-representations for our running example.

Example 2 ( $\mathcal{R}_{\text {bird }}$, cont.) The verification/falsification behavior of the worlds and conditionals for $\mathcal{R}_{\text {bird }}$ is shown in the left-hand side of Table 2 . Taking this verification/falsification behavior into account, the $\operatorname{CSP} C R\left(\mathcal{R}_{\text {bird }}\right)$ is given by:

$$
\begin{aligned}
& \eta_{i} \geq 0 \quad \text { for } 1 \leq i \leq 4 \\
& \eta_{1}>\min \left\{\eta_{2}+\eta_{3}, 0\right\} \\
& \eta_{2}>\min \left\{\eta_{1}, \eta_{4}\right\}-\min \left\{\eta_{3}, \eta_{4}\right\} \\
& \eta_{3}>\eta_{1}-\eta_{2} \\
& \eta_{4}>\min \left\{\eta_{2}+\eta_{3}, \eta_{1}\right\}-\min \left\{\eta_{2}, 0\right\}
\end{aligned}
$$


Table 2 Schema for c-representations for the knowledge base in Example 2: verification (v), falsification (f), not applicable (-), impacts $\left(\eta_{i}\right)$, six solution vectors $\vec{\eta}_{1}, \ldots, \vec{\eta}_{6}$, and their induced c-representations $\kappa \vec{\eta}_{1}, \ldots, \kappa \vec{\eta}_{6}$ for $\mathcal{R}_{\text {bird }}$

\begin{tabular}{|c|c|c|c|c|c|c|c|c|c|c|c|}
\hline$\omega$ & $\begin{array}{c}r_{1}: \\
(f \mid b)\end{array}$ & $\begin{array}{c}r_{2}: \\
(\bar{f} \mid p)\end{array}$ & $\begin{array}{c}r_{3}: \\
(\bar{f} \mid p b)\end{array}$ & $\begin{array}{c}r_{4}: \\
(b \mid p)\end{array}$ & $\begin{array}{c}\text { impact } \\
\text { on } \omega\end{array}$ & $\begin{array}{r}\kappa \vec{\eta}_{1} \\
(\omega)\end{array}$ & $\begin{array}{r}\kappa \vec{\eta}_{2} \\
(\omega)\end{array}$ & $\begin{array}{r}\kappa \vec{\eta}_{3} \\
(\omega)\end{array}$ & $\begin{array}{c}\kappa \vec{\eta}_{4} \\
(\omega)\end{array}$ & $\begin{array}{r}\kappa \vec{\eta}_{5} \\
(\omega)\end{array}$ & $\begin{array}{r}\kappa \vec{\eta}_{6} \\
(\omega)\end{array}$ \\
\hline$p b f$ & v & $f$ & $f$ & $v$ & $\eta_{2}+\eta_{3}$ & 2 & 2 & 2 & 4 & 7 & 6 \\
\hline$p b \bar{f}$ & $f$ & v & v & v & $\eta_{1}$ & 1 & 1 & 1 & 1 & 2 & 3 \\
\hline$p \bar{b} f$ & - & $f$ & - & $f$ & $\eta_{2}+\eta_{4}$ & 4 & 2 & 3 & 4 & 9 & 6 \\
\hline$p \bar{b} \bar{f}$ & - & v & - & $f$ & $\eta_{4}$ & 2 & 2 & 2 & 2 & 6 & 6 \\
\hline $\bar{p} b f$ & $v$ & - & - & - & 0 & 0 & 0 & 0 & 0 & 0 & 0 \\
\hline $\bar{p} b \bar{f}$ & $f$ & - & - & - & $\eta_{1}$ & 1 & 1 & 1 & 1 & 2 & 3 \\
\hline $\bar{p} \bar{b} f$ & - & - & - & - & 0 & 0 & 0 & 0 & 0 & 0 & 0 \\
\hline $\bar{p} \bar{b} \bar{f}$ & - & - & - & - & 0 & 0 & 0 & 0 & 0 & 0 & 0 \\
\hline impacts: & $\eta_{1}$ & $\eta_{2}$ & $\eta_{3}$ & $\eta_{4}$ & & & & & & & \\
\hline$\vec{\eta}_{1}$ & 1 & 2 & 0 & 2 & & & & & & & \\
\hline$\vec{\eta}_{2}$ & 1 & 0 & 2 & 2 & & & & & & & \\
\hline$\vec{\eta}_{3}$ & 1 & 1 & 1 & 2 & & & & & & & \\
\hline$\vec{\eta}_{4}$ & 1 & 2 & 2 & 2 & & & & & & & \\
\hline$\vec{\eta}_{5}$ & 2 & 3 & 4 & 6 & & & & & & & \\
\hline$\vec{\eta}_{6}$ & 3 & 0 & 6 & 6 & & & & & & & \\
\hline
\end{tabular}

For illustration, in the lower part of Table 2 , some solution vectors $\vec{\eta}_{1}, \ldots, \vec{\eta}_{6} \in$ $\operatorname{Sol}\left(C R\left(\mathcal{R}_{\text {bird }}\right)\right)$ are shown, along with their induced c-representations $\kappa \vec{\eta}_{1}, \ldots, \kappa \vec{\eta}_{6} \in$ $\mathcal{O}\left(C R\left(\mathcal{R}_{\text {bird }}\right)\right)$ given in the right-hand side of Table 2.

As every ranking model of $\mathcal{R}$, each c-representation $\kappa \vec{\eta}$ gives rise to an inference relation according to (3). While for each consistent $\mathcal{R}$, the system $Z$ ranking function $\kappa^{Z}$ is uniquely determined, there may be many different c-representations of $\mathcal{R}$. While system $\mathrm{P}$ inference considers all ranking models of $\mathcal{R}$, c-inference is an inference relation taking all c-representations of $\mathcal{R}$ into account.

Definition 4 (c-inference, $\boldsymbol{\sim}_{\mathcal{R}}^{c}[2,4]$ ) Let $\mathcal{R}$ be a knowledge base and let $A, B$ be formulas. $B$ is a (skeptical) c-inference from $A$ in the context of $\mathcal{R}$, denoted by $A \sim_{\mathcal{R}}^{c} B$, iff $A \sim{ }^{\kappa} B$ holds for all c-representations $\kappa$ for $\mathcal{R}$.

Example 3 ( $\mathcal{R}_{\text {bird }}$, cont.) Given the knowledge base $\mathcal{R}_{\text {bird }}$, for the system $\mathrm{Z}$ inference that a bird which is not a penguin usually flies, i.e, $\bar{p} b \sim{\underset{\mathcal{R}}{\text { bird }}}_{\text {ind }}^{Z} f$, considered in Example 1, we also have $\bar{p} b \sim^{\kappa} \vec{\eta}_{i} f$ for every c-representation $\kappa \vec{\eta}_{i}$ from Example 2. Furthermore, we also have the c-inference $\bar{p} b \sim{ }_{\mathcal{R}_{\text {bird }}}^{c} f$ because $\bar{p} b \sim^{\kappa} f$ holds for every c-representation $\kappa$ of $\mathcal{R}_{\text {bird }}$.

Another example of a c-inference in the context of $\mathcal{R}_{\text {bird }}$ is that a flying bird is usually not a penguin, i.e., that $b f \sim{ }_{\mathcal{R}_{\text {bird }}}^{c} \bar{p}$ holds.

In addition to (skeptical) c-inference, also other modes of inference based on crepresentations have been proposed [3]. For two formulas $A$ and $B, B$ is a credulous 
c-inference from $A$ in the context of $\mathcal{R}$, if there is a c-representation $\kappa$ accepting $\mathcal{R}$ such that $A \sim{ }^{\kappa} B$. Furthermore, $B$ is a weakly skeptical c-inference from $A$ in the context of $\mathcal{R}$ if there is a c-representation $\kappa$ for $\mathcal{R}$ such that $A \sim^{\kappa} B$ and there is no c-representation $\kappa^{\prime}$ for $\mathcal{R}$ such that $A \sim{ }^{\kappa^{\prime}} \bar{B}$. For a detailed study of the properties and interrelationships of the different modes of c-inference we refer the reader to [5]; in this article, we will focus on (skeptical) c-inference.

\section{Resource bounded c-inference}

We will now investigate how to answer the question whether a c-inference $A \sim_{\mathcal{R}}^{c} B$ holds by taking only a subset of all c-representations of $\mathcal{R}$ into account. If a (non-empty) knowledge base $\mathcal{R}$ is consistent, there are infinitely many different c-representations accepting $\mathcal{R}$, and obviously, if for two c-representations $\kappa, \kappa^{\prime}$ we have $A \sim{ }^{\kappa} B$ if and only if $A \sim{ }^{\kappa^{\prime}} B$ for all formulas $A, B$, then it suffices to consider only one of the ranking models $\kappa, \kappa^{\prime}$. For characterizing a subset of all c-representations of a knowledge base $\mathcal{R}$, an upper bound for the impact values inducing the c-representations of $\mathcal{R}$ can be used, yielding a finite domain constraint satisfaction problem.

Definition $5\left(\boldsymbol{C R}{ }^{u}(\mathcal{R})[4]\right)$ Let $\mathcal{R}=\left\{\left(B_{1} \mid A_{1}\right), \ldots,\left(B_{n} \mid A_{n}\right)\right\}$ and $u \in \mathbb{N}$. The finite domain constraint satisfaction problem $C R^{u}(\mathcal{R})$ on the constraint variables $\left\{\eta_{1}, \ldots, \eta_{n}\right\}$ ranging over $\mathbb{N}_{0}$ is given by the conjunction of the constraints, for all $i \in\{1, \ldots, n\}$ :

$$
\begin{aligned}
& \eta_{i} \geq 0 \\
& \eta_{i}>\min _{\omega \models A_{i} B_{i}} \sum_{\substack{j \neq i \\
\omega \models A_{j} \overline{B_{j}}}} \eta_{j}-\min _{\omega \models A_{i} \bar{B}_{i}} \sum_{\substack{j \neq i \\
\omega \models A_{j} \overline{B_{j}}}} \eta_{j} \\
& \eta_{i} \leq u
\end{aligned}
$$

A solution of $C R^{u}(\mathcal{R})$ is an $n$-tuple $\left(\eta_{1}, \ldots, \eta_{n}\right) \in \mathbb{N}_{0}^{n}$, its set of solutions is denoted by $\operatorname{Sol}\left(C R^{u}(\mathcal{R})\right)$. For $\vec{\eta} \in \operatorname{Sol}\left(C R^{u}(\mathcal{R})\right)$ and $\kappa$ as in $(6), \kappa$ is the OCF induced by $\vec{\eta}$, denoted by $\kappa \vec{\eta}$, and the set of all induced OCFs is denoted by $\mathcal{O}\left(C R^{u}(\mathcal{R})\right)=\{\kappa \vec{\eta} \mid \vec{\eta} \in$ $\left.\operatorname{Sol}\left(C R^{u}(\mathcal{R})\right)\right\}$.

Example 4 ( $\mathcal{R}_{\text {bird }}$, cont.) Given the c-representations considered in Example 2, out of the solutions $\vec{\eta}_{1}, \ldots, \vec{\eta}_{6} \in \operatorname{Sol}\left(C R\left(\mathcal{R}_{\text {bird }}\right)\right)$ only $\vec{\eta}_{1}, \vec{\eta}_{2}, \vec{\eta}_{3}$, and $\vec{\eta}_{4}$ are elements of $\operatorname{Sol}\left(C R^{2}\left(\mathcal{R}_{\text {bird }}\right)\right)$, while $\vec{\eta}_{5}$ and $\vec{\eta}_{6}$ are in $\operatorname{Sol}\left(C R^{u}\left(\mathcal{R}_{\text {bird }}\right)\right)$ only if $u \geq 6$.

InfOCF-Web [27] is a tool for reasoning with conditional knowledge bases whose objective is to provide an easy-to-use online tool for computing and comparing a variety of different inference relations induced by a knowledge base. Using the functionalities of InfOCF-Web, we illustrate the effect of using the upper bound $u$ on cutting down the number of solutions of the resulting CSP.

Example 5 ( $\mathcal{R}_{\text {bird }}$, cont.) While $\operatorname{Sol}\left(C R\left(\mathcal{R}_{\text {bird }}\right)\right)$ contains infinitely many different solutions, $\operatorname{Sol}\left(C R^{u}\left(\mathcal{R}_{\text {bird }}\right)\right)$ has finitely many elements for every $u \in \mathbb{N}$. Employing InfOCF-Web for computing the set $\operatorname{Sol}\left(C R^{u}\left(\mathcal{R}_{\text {bird }}\right)\right)$ for various values of $u$, reveals that, for instance, $\operatorname{Sol}\left(C R^{10}\left(\mathcal{R}_{\text {bird }}\right)\right)$ has 4.740 elements, $\operatorname{Sol}\left(C R^{6}\left(\mathcal{R}_{\text {bird }}\right)\right)$ has 615 elements, $\operatorname{Sol}\left(C R^{3}\left(\mathcal{R}_{\text {bird }}\right)\right)$ has 36 elements, and $\operatorname{Sol}\left(C R^{2}\left(\mathcal{R}_{\text {bird }}\right)\right)$ has only 6 elements. 
Instead of taking all c-representations into account as it is done for c-inference according to Definition 4, c-inference defined with respect to a maximal impact value can be viewed as a kind of resource-bounded inference operation.

Definition 6 (c-inference under maximal impact value, $\boldsymbol{}_{\mathcal{R}}^{c, u}[4]$ ) Let $\mathcal{R}$ be a knowledge base, $u \in \mathbb{N}$, and let $A, B$ be formulas. $B$ is a (skeptical) $c$-inference from $A$ in the context of $\mathcal{R}$ under maximal impact value $u$, denoted by $A \sim_{\mathcal{R}}^{c, u} B$, iff $A \sim^{\kappa} B$ holds for all c-representations $\kappa$ with $\kappa \in \mathcal{O}\left(C R^{u}(\mathcal{R})\right)$.

The following definition introduces a criterion for a maximal impact value ensuring that $\sim_{\mathcal{R}}^{c, u}$ fully realizes skeptical c-inference. For an OCF $\kappa$, the definition uses the total preorder $\preccurlyeq_{\kappa}$ on worlds given by $\omega_{1} \preccurlyeq_{\kappa} \omega_{2}$ iff $\kappa\left(\omega_{1}\right) \leq \kappa\left(\omega_{2}\right)$.

Definition 7 (regular, minimally regular $[4,8]$ ) For $\mathcal{R}$ let $\hat{u} \in \mathbb{N}$ be the smallest number such that $\left|\left\{\preccurlyeq_{\kappa} \mid \kappa \in \mathcal{O}\left(C R^{\hat{u}}(\mathcal{R})\right)\right\}\right|=\mid\left\{\preccurlyeq_{\kappa}|\kappa \in \mathcal{O}(C R(\mathcal{R}))|\right.$. Then $C R^{u}(\mathcal{R})$ is called regular iff $u \geq \hat{u}$, and $C R^{\hat{u}}(\mathcal{R})$ is minimally regular; we also say that $u$ is regular for $\mathcal{R}$ and $\hat{u}$ is minimally regular for $\mathcal{R}$.

While $C R(\mathcal{R})$ correctly and completely models the set of all c-representations for $\mathcal{R}$ (Proposition 1), every regular $C R^{u}(\mathcal{R})$ is sound and complete when taking inferential equivalence into account.

Definition $8\left(\equiv_{\uparrow}\right)$ Two ranking functions $\kappa, \kappa^{\prime}$ are inferentially equivalent, denoted by $\kappa \equiv_{\vdash} \kappa^{\prime}$ iff for all $(B \mid A)$ it is the case that $\kappa \models(B \mid A)$ iff $\kappa^{\prime} \models(B \mid A)$.

For instance, if there is a $k \in \mathbb{N}$ such that $\kappa^{\prime}(\omega)=k \cdot \kappa(\omega)$ for all worlds $\omega$, then $\kappa \equiv_{\kappa} \kappa^{\prime}$; in general, two ranking functions are inferentially equivalent iff they induce the same total preorder on worlds.

Proposition 2 [4] For ranking functions $\kappa$ and $\kappa^{\prime}$, we have $\kappa \equiv_{\sim} \kappa^{\prime}$ iff for all $\omega_{1}, \omega_{2} \in \Omega$ it is the case that $\kappa\left(\omega_{1}\right) \leq \kappa\left(\omega_{2}\right)$ iff $\kappa^{\prime}\left(\omega_{1}\right) \leq \kappa^{\prime}\left(\omega_{2}\right)$.

For a set $M$ and an equivalence relation $\equiv$ on $M$, the set of equivalence classes induced by $\equiv$ is denoted by $M_{/ \equiv}$. According to Definition 7, regularity of $u$ for a knowledge base $\mathcal{R}$

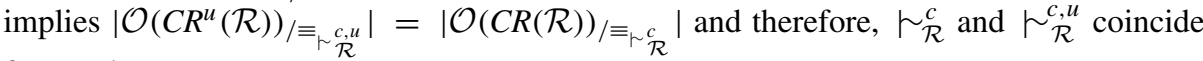
for regular $u$.

Proposition 3 [4] Let $\mathcal{R}$ be a knowledge base, $C R^{u}(\mathcal{R})$ regular, and $A, B$ be formulas. Then $A \sim_{\mathcal{R}}^{c} B$ iff $A \sim_{\mathcal{R}}^{c, u} B$.

When we are not interested in capturing all c-representations as done by a regular $C R^{u}(\mathcal{R})$, but aim at capturing c-inference instead, we can specify a maximal impact value from this inference perspective.

Definition 9 (sufficient, minimally sufficient $[4,8]$ ) Let $\mathcal{R}$ be a knowledge base and let $u \in \mathbb{N}$. Then $C R^{u}(\mathcal{R})$ is called sufficient iff for all formulas $A, B$ we have

$$
A \sim_{\mathcal{R}}^{c} B \text { iff } A \sim_{\mathcal{R}}^{c, u} B
$$


If $C R^{u}(\mathcal{R})$ is sufficient, we will also call $u$ sufficient for $\mathcal{R}$. If $\hat{u}$ is sufficient for $\mathcal{R}$ and $\hat{u}-1$ is not sufficient for $\mathcal{R}$, then $\hat{u}$ is minimally sufficient for $\mathcal{R}$.

The condition that $C R^{l}(\mathcal{R})$ is regular is only a sufficient condition for (14) but not necessary.

Proposition 4 (regular vs. sufficient [8]) Let $\mathcal{R}$ be consistent and $u \in \mathbb{N}$. If $C R^{u}(\mathcal{R})$ is regular, then it is also sufficient; the reverse is not true in general.

If $C R^{u}(\mathcal{R})$ is regular, it is also sufficient according to Proposition 3. For showing the other direction, the knowledge base $\mathcal{R}_{2}=\left\{\left(a_{1} \mid \top\right),\left(a_{2} \mid \top\right)\right\}$ over the signature $\Sigma=\left\{a_{1}, a_{2}\right\}$ is used in [8]. The verification and falsification behavior as well as all solution vectors and their induced ranking models for the CSP $C R^{2}\left(\mathcal{R}_{2}\right)$ are given in Table 3. The maximal impact value $u=2$ is regular for $\mathcal{R}_{2}$, and for the set $\operatorname{Sol}\left(C R^{2}\left(\mathcal{R}_{2}\right)\right)$ consisting of the four elements

$$
\vec{\eta}_{1}=(1,1), \vec{\eta}_{2}=(1,2), \vec{\eta}_{3}=(2,1), \vec{\eta}_{4}=(2,2)
$$

the following observations hold: Since $\vec{\eta}_{4}$ is a multiple of $\vec{\eta}_{1}$, the inference relations associated with their induced ranking functions $\kappa \vec{\eta}_{4}$ and $\kappa \vec{\eta}_{1}$ are inferentially equivalent. For the reduced maximal impact value $u=1$, obviously, $C R^{1}\left(\mathcal{R}_{2}\right)$ only has $\vec{\eta}_{1}$ as a solution. With respect to the solutions of $C R^{2}\left(\mathcal{R}_{2}\right)$, every inference that requires a different ranking of the worlds $a_{1} \overline{a_{2}}$ and $\overline{a_{1}} a_{2}$ only holds when considering either $\kappa \vec{\eta}_{2}$ or $\kappa \vec{\eta}_{3}$, but not when taking also $\kappa \vec{\eta}_{1}$ into account. Hence, such inferences where the worlds $a_{1} \overline{a_{2}}$ and $\overline{a_{1}} a_{2}$ must be ranked differently do not hold under (skeptical) inference over $\left\{\kappa \vec{\eta}_{1}, \kappa \vec{\eta}_{2}, \kappa \vec{\eta}_{3}\right\}$. On the other hand, every inference that holds in $\kappa \vec{\eta}_{1}$ also holds in $\kappa \vec{\eta}_{2}$ and $\kappa \vec{\eta}_{3}$. Therefore, $C R^{1}\left(\mathcal{R}_{1}\right)$ is sufficient but not regular. Furthermore, while $C R^{2}\left(\mathcal{R}_{2}\right)$ is minimally regular, $C R^{1}\left(\mathcal{R}_{2}\right)$ is minimally sufficient.

Thus, for answering the question whether $A \sim_{\mathcal{R}}^{c} B$ holds, we can check $A \sim_{\mathcal{R}}^{c, u} B$ instead if $u$ is sufficient or minimally sufficient. While it has been conjectured that in general the number of conditionals $u=|\mathcal{R}|$ in the given knowledge base is sufficient [8], in the following sections we will show that this is not the case.

Table 3 Verification (v), falsification (f), impacts $\left(\eta_{i}\right)$, solution vectors $\left(\vec{\eta}_{i}\right)$, and induced OCFs $\left(\kappa \vec{\eta}_{i}\right)$ for $C R^{2}\left(\mathcal{R}_{2}\right)$

\begin{tabular}{|c|c|c|c|c|c|c|c|}
\hline$\omega$ & $\begin{array}{c}r_{1}: \\
\left(a_{1} \mid \top\right)\end{array}$ & $\begin{array}{c}r_{2}: \\
\left(a_{2} \mid \top\right)\end{array}$ & $\begin{array}{c}\text { impact } \\
\text { on } \omega\end{array}$ & $\begin{array}{c}\kappa \vec{\eta}_{1} \\
(\omega)\end{array}$ & $\begin{array}{c}\kappa \vec{\eta}_{2} \\
(\omega)\end{array}$ & $\begin{array}{c}\kappa \vec{\eta}_{3} \\
(\omega)\end{array}$ & $\begin{array}{c}\kappa \vec{\eta}_{4} \\
(\omega)\end{array}$ \\
\hline$a_{1} a_{2}$ & v & v & 0 & 0 & 0 & 0 & 0 \\
\hline$a_{1} \overline{a_{2}}$ & v & $f$ & $\eta_{2}$ & 1 & 2 & 1 & 2 \\
\hline$\overline{a_{1}} a_{2}$ & $f$ & $\mathrm{v}$ & $\eta_{1}$ & 1 & 1 & 2 & 2 \\
\hline$\overline{a_{1}} \overline{a_{2}}$ & $f$ & $f$ & $\eta_{1}+\eta_{2}$ & 2 & 3 & 3 & 4 \\
\hline impacts: & $\eta_{1}$ & $\eta_{2}$ & & & & & \\
\hline$\vec{\eta}_{1}$ & 1 & 1 & & & & & \\
\hline$\vec{\eta}_{2}$ & 1 & 2 & & & & & \\
\hline$\vec{\eta}_{3}$ & 2 & 1 & & & & & \\
\hline$\vec{\eta}_{4}$ & 2 & 2 & & & & & \\
\hline
\end{tabular}


Other forms of resource-bounded c-inference restrict the class of c-representations to be taken into account by employing a notion of preferred models. Such preferred models could be obtained by applying the concept of minimal specificity (see e.g. [12]). For crepresentations, an option for this is to take only minimal impact vectors into account; for instance, the set of Pareto-minimal solutions of $C R\left(\mathcal{R}_{\text {bird }}\right)$ is given by the three vectors $\left\{\vec{\eta}_{1}, \vec{\eta}_{2}, \vec{\eta}_{3}\right\}$ presented in Table 2 . Detailed information about c-inference with respect to preferred minimal models can be found in [5].

\section{A restricted constraint satisfaction problem}

While the constraint system $C R(\mathcal{R})$ is sound and complete with respect to the set of crepresentations of $\mathcal{R}$, solving $C R(\mathcal{R})$ requires to take the complex constraints in the CSP into account. For deriving properties of $C R(\mathcal{R})$ and its space of solutions, a simpler form of the CSP would be helpful. Towards this aims, we will introduce a simplified CSP in this section called $C R_{o p}(\mathcal{R})$. Unlike $C R(\mathcal{R})$, which depends on the full verification/ falsification behaviour of the conditionals in $\mathcal{R}, C R_{O p}(\mathcal{R})$ depends on the simpler structure of the ordered partition $O P(\mathcal{R})$. In contrast to solving $C R(\mathcal{R})$, the set of solutions to this CSP can easily be constructed in a linear number of steps, and we will show that every solution to $C R_{o p}(\mathcal{R})$ is also a solution to $C R(\mathcal{R})$. Let us start with the formal definition.

Definition $10\left(\boldsymbol{C R}_{\boldsymbol{o p}}(\mathcal{R})\right)$ Let $\mathcal{R}=\left\{r_{i}=\left(B_{i} \mid A_{i}\right), i=1, \ldots, n\right\}$ be a consistent knowledge base with inclusion-maximal partition $O P(\mathcal{R})=\left(\mathcal{R}_{0}, \ldots, \mathcal{R}_{k}\right)$. The restricted constraint satisfaction problem for c-representations of $\mathcal{R}$, denoted by $C R_{o p}(\mathcal{R})$, on the constraint variables $\left\{\eta_{1}, \ldots, \eta_{n}\right\}$ ranging over $\mathbb{N}_{0}$ is given by the conjunction of the constraints, for all $i \in\{1, \ldots, n\}$ :

$$
\eta_{i}>\sum_{\substack{j \in\{1, \ldots, n\} \\ r_{j} \in \bigcup_{l=0}^{m-1} \mathcal{R}_{l}}} \eta_{j} \quad \text { if } r_{i} \in \mathcal{R}_{0},
$$

In the following remark, we provide an equivalent, but more compact representation of $C R_{o p}(\mathcal{R})$ which we will often use in the rest of this article.

Remark 1 Using $\bigcup_{l=0}^{m-1} \mathcal{R}_{l}=\varnothing$ for $m=0$, we can equivalently write (15), (16) in the compact notation

$$
\eta_{i}>\sum_{\substack{j \in\{1, \ldots, n\} \\ r_{j} \in \bigcup_{l=0}^{m-1} \mathcal{R}_{l}}} \eta_{j}
$$

for all $i \in\{1, \ldots, n\}$ where $m=m(i) \in\{0, \ldots, k\}$ in (17) denotes the unique integer with $r_{i} \in \mathcal{R}_{m}$.

In contrast to $C R(\mathcal{R})$, we have that $C R_{o p}(\mathcal{R})$ indeed depends only on the inclusionmaximal partition $\operatorname{OP}(\mathcal{R})=\left(\mathcal{R}_{0}, \ldots, \mathcal{R}_{k}\right)$ of $\mathcal{R}$, but not on the details of the verification/ falsification behavior of $\mathcal{R}$ as expressed in the right hand side of the constraint (8). This results in a simpler structure of $C R_{o p}(\mathcal{R})$ compared to $C R(\mathcal{R})$. Especially we see that the right hand side of (16) only consists of positive summands instead of positive and negative summands as in the right hand side of (8). 
Example $6\left(C R_{o p}(\mathcal{R})\right)$ To illustrate the structure of $C R_{o p}(\mathcal{R})$ consider a knowledge base $\mathcal{R}=\left\{r_{i} \mid i=1, \ldots, 7\right\}$ where the inclusion-maximal ordered partition is given by $O P(\mathcal{R})=\left(\mathcal{R}_{0}, \mathcal{R}_{1}, \mathcal{R}_{2}\right)$ with

$$
\begin{aligned}
& \mathcal{R}_{0}=\left\{r_{1}, r_{2}\right\}, \\
& \mathcal{R}_{1}=\left\{r_{3}, r_{4}, r_{5}\right\}, \\
& \mathcal{R}_{2}=\left\{r_{6}, r_{7}\right\} .
\end{aligned}
$$

Then we obtain for $C R_{o p}(\mathcal{R})$ the conjunction of the constraints

$$
\begin{array}{lrl}
\eta_{i}>0 & \text { for } i \in\{1,2\}, \\
\eta_{i}>\sum_{j=1}^{2} \eta_{j} & \text { for } i \in\{3,4,5\}, \\
\eta_{i}>\sum_{j=1}^{5} \eta_{j} & \text { for } i \in\{6,7\}
\end{array}
$$

for the constraint variables $\left\{\eta_{1}, \ldots, \eta_{7}\right\}$. This example illustrates that $C R_{o p}(\mathcal{R})$ is completely determined by the partition $O P(\mathcal{R})=\left(\mathcal{R}_{0}, \mathcal{R}_{1}, \mathcal{R}_{2}\right)$ of $\mathcal{R}$ and therefore, in contrast to $C R(\mathcal{R})$, does not depend on the details of the verification/falsification behavior of the knowledge base $\mathcal{R}$.

Let us illustrate $C R_{o p}(\mathcal{R})$ also for our running example.

Example $7\left(C R_{o p}\left(\mathcal{R}_{\text {bird }}\right)\right)$ For the knowledge base $\mathcal{R}_{\text {bird }}$ from Example 1, the inclusionmaximal partition is $O P\left(\mathcal{R}_{\text {bird }}\right)=\left(\mathcal{R}_{0}, \mathcal{R}_{1}\right)$ with

$$
\begin{aligned}
& \mathcal{R}_{0}=\left\{r_{1}=(f \mid b)\right\}, \\
& \mathcal{R}_{1}=\left\{r_{2}=(\bar{f} \mid p), r_{3}=(\bar{f} \mid b p), r_{4}=(b \mid p)\right\} .
\end{aligned}
$$

In contrast to $C R\left(\mathcal{R}_{\text {bird }}\right)$ which is given in Example 2, $C R_{o p}\left(\mathcal{R}_{\text {bird }}\right)$ consists of just some very simple inequalities:

$$
\begin{aligned}
& \eta_{1}>0, \\
& \eta_{2}>\eta_{1}, \\
& \eta_{3}>\eta_{1}, \\
& \eta_{4}>\eta_{1} .
\end{aligned}
$$

In general, its simpler structure causes $C R_{o p}(\mathcal{R})$ not to be equivalent to $C R(\mathcal{R})$. However, the following proposition shows that $C R_{o p}(\mathcal{R})$ refines $C R(\mathcal{R})$ so that every solution to $C R_{o p}(\mathcal{R})$ is also a solution to $C R(\mathcal{R})$. This proposition will be exploited later on when establishing the exponential lower bound for the maximal impact factor to fully realize (skeptical) c-inference.

Proposition $5\left(C R_{o p}(\mathcal{R})\right.$ refines $\left.C R(\mathcal{R})\right)$ Let $\mathcal{R}=\left\{r_{i}=\left(B_{i} \mid A_{i}\right) \mid i=1, \ldots, n\right\}$ be a consistent knowledge base with inclusion-maximal partition $\operatorname{OP}(\mathcal{R})=\left(\mathcal{R}_{0}, \ldots, \mathcal{R}_{k}\right)$. Then $\operatorname{Sol}\left(C R_{o p}(\mathcal{R})\right) \subseteq \operatorname{Sol}(C R(\mathcal{R}))$.

Proof Consider a solution $\vec{\eta}=\left(\eta_{1}, \ldots, \eta_{n}\right)$ to $C R_{o p}(\mathcal{R})$. To prove $\vec{\eta} \in \operatorname{Sol}(C R(\mathcal{R}))$ it suffices, due to Proposition 1, to show that $\kappa \vec{\eta}$ given by (6) is a c-representation. Consequently 
we have to show $\kappa \vec{\eta}\left(A_{i} B_{i}\right)<\kappa \vec{\eta}\left(A_{i} \bar{B}_{i}\right)$ for all $i \in\{1, \ldots, n\}$. Let us fix $i \in\{1, \ldots, n\}$ and let $m=m(i) \in\{0, \ldots, k\}$ such that $r_{i} \in \mathcal{R}_{m}$.

Step 1. Since $\left(\mathcal{R}_{0}, \ldots, \mathcal{R}_{k}\right)$ is an inclusion-maximal partition there exists $\omega^{\prime} \in \Omega$ verifying $A_{i} B_{i}$ and not falsifying any conditional from $\bigcup_{l=m}^{k} \mathcal{R}_{l}$. We obtain

$$
\begin{aligned}
& \kappa \vec{\eta}\left(\omega^{\prime}\right)=\sum_{\substack{j \in\{1, \ldots, n\} \\
\omega^{\prime} \models A_{j} \bar{B}_{j}}} \eta_{j} \\
& =\sum_{\substack{j \in\{1, \ldots, n\} \\
r_{j} \in \bigcup_{l=0}^{m-1} \mathcal{R}_{l}, \omega^{\prime} \models A_{j} \overline{B_{j}}}} \eta_{j} \\
& \begin{array}{l}
\leq \sum_{\substack{j \in\{1, \ldots, n\} \\
r_{j} \in \bigcup_{l=0}^{m-1} \mathcal{R}_{l}}}^{<\eta_{j}} \\
<\eta_{i}
\end{array}
\end{aligned}
$$

Thus the definition of $\kappa\left(A_{i} B_{i}\right)$ and (18) yield

$$
\kappa \vec{\eta}\left(A_{i} B_{i}\right)=\min _{\omega \models A_{i} B_{i}} \kappa \vec{\eta}(w) \leq \kappa \vec{\eta}\left(\omega^{\prime}\right)<\eta_{i} .
$$

Step 2. We obtain

$$
\kappa \vec{\eta}\left(A_{i} \overline{B_{i}}\right)=\min _{\substack{\omega \models A_{i} \overline{B_{i}}}} \sum_{\substack{j \in\{1, \ldots, n\} \\ \omega \models A_{j} \overline{B_{j}}}} \eta_{j} \geq \eta_{i} .
$$

Combining (19), (20) yields $\kappa_{\vec{\eta}}\left(A_{i} B_{i}\right)<\kappa_{\vec{\eta}}\left(A_{i} \bar{B}_{i}\right)$, finishing the proof of $\kappa_{\vec{\eta}} \in$ $\mathcal{O}(C R(\mathcal{R}))$.

The next proposition tells us how a solution of $C R_{o p}(\mathcal{R})$ can be obtained in a straightforward way.

Proposition 6 Let $\mathcal{R}=\left\{r_{i}=\left(B_{i} \mid A_{i}\right), i=1, \ldots, n\right\}$ be a consistent knowledge base with inclusion-maximal partition $\operatorname{OP}(\mathcal{R})=\left(\mathcal{R}_{0}, \ldots, \mathcal{R}_{k}\right)$. Then $\left(\eta_{1}, \ldots, \eta_{n}\right)$ well-defined by

$$
\eta_{i}=1+\sum_{\substack{j \in\{1, \ldots, n\} \\ r_{j} \in \bigcup_{l=0}^{m-1} \mathcal{R}_{l}}} \eta_{j}
$$

for all $i \in\{1, \ldots, n\}$ where $m=m(i) \in\{0, \ldots, k\}$ with $r_{i} \in \mathcal{R}_{m}$ is a solution to $C R(\mathcal{R})$.

Proof Looking at (17) we obtain that $\vec{\eta}=\left(\eta_{1}, \ldots, \eta_{n}\right)$ defined by (21) is a solution to $C R_{o p}(\mathcal{R})$. An application of Proposition 5 shows $\vec{\eta} \in \operatorname{Sol}(C R(\mathcal{R}))$.

Note that Proposition 6 implies that for every consistent $\mathcal{R}$, the solution $\vec{\eta}=$ $\left(\eta_{1}, \ldots, \eta_{n}\right)$ given by $(21)$ is easily obtained from the inclusion-maximal ordered partition $O P(\mathcal{R})$ of $\mathcal{R}$. Given $O P(\mathcal{R})$, just $n$ simple steps are required to determine $\left(\eta_{1}, \ldots, \eta_{n}\right)$.

Example $8\left(\mathcal{R}_{\text {bird }}\right.$, cont.) Consider the system $C R_{\text {op }}\left(\mathcal{R}_{\text {bird }}\right)$ given in Example 7 for the knowledge base $\mathcal{R}_{\text {bird }}$ from Example 1 . Constructing a solution $\vec{\eta}=\left\{\eta_{1}, \eta_{2}, \eta_{3}, \eta_{4}\right\}$ of $C R_{o p}\left(\mathcal{R}_{\text {bird }}\right)$ according to (21), assigns to the impact factor $\eta_{1}$ corresponding to the conditional from $\mathcal{R}_{0}$ the value 1 , and it assigns to the impact factors $\eta_{2}, \eta_{3}$, and $\eta_{4}$, corresponding to the more specialized conditionals from $\mathcal{R}_{1}$, the higher value 2 , yielding $\vec{\eta}=(1,2,2,2)$. Due to Proposition 6, we have that $\vec{\eta} \in \operatorname{Sol}\left(C R\left(\mathcal{R}_{\text {bird }}\right)\right)$. Note that $\vec{\eta}$ coincides with $\vec{\eta}_{4}$ from Table 2; thus, its induced c-representation is $\kappa \vec{\eta}_{4}$, also shown in Table 2. 


\section{A criterion such that $C R^{n-1}(\mathcal{R})$ is sufficient}

An important topic is to provide criteria for a knowledge base $\mathcal{R}$ to obtain explicit bounds for the maximal impact factor $u$ such that $C R^{u}(\mathcal{R})$ is sufficient. The following example presents a knowledge base $\mathcal{R}$ such that the linear bound $u=|\mathcal{R}|-1$ is sufficient.

Example 9 In [9] the knowledge base $\mathcal{R}_{n}=\left\{\left(a_{1} \mid \top\right), \ldots,\left(a_{n} \mid \top\right)\right\}$ of $n$ conditionals facts over $\Sigma_{n}=\left\{a_{1}, \ldots, a_{n}\right\}$ is introduced. In [9, Proposition 19] the authors show that $C R^{n-1}\left(\mathcal{R}_{n}\right)$ is sufficient for $n>1$. Thus, for all formulas $A, B$ over $\Sigma_{n}$ the entailment $A \sim{ }_{\mathcal{R}_{n}}^{c} B$ holds iff $A \sim_{\mathcal{R}_{n}}^{c, n-1} B$ holds.

Motivated by this example, we want to present a general criterion for a knowledge base $\mathcal{R}$ to obtain a linear bound for the maximal impact factor $u$ such that $C R^{u}(\mathcal{R})$ is sufficient. In more detail, the objective of this section is to prove for a knowledge base $\mathcal{R}=\left\{r_{1}, \ldots, r_{n}\right\}$ with a world $\omega \in \Omega$ verifying all conditionals from $\mathcal{R}$ we have that $C R^{n-1}(\mathcal{R})$ is sufficient.

Proposition 7 Let $n \in \mathbb{N}, n>1$, and let $\mathcal{R}=\left\{\left(B_{i} \mid A_{i}\right), i=1, \ldots, n\right\}$ be a knowledge base. If there exists $\omega \in \Omega$ with $\omega \models A_{i} B_{i}$ for all $i \in\{1, \ldots n\}$, then the knowledge base is consistent and $C R^{n-1}(\mathcal{R})$ is sufficient.

Proof Choose $\tilde{\omega} \in \Omega$ accepting all conditionals from $\mathcal{R}$. Then

$$
\begin{aligned}
& \min _{\omega \models A_{i} B_{i}} \sum_{\substack{j \neq i \\
\omega \models A_{j} \overline{B_{j}}}} \eta_{j}-\min _{\omega \models A_{i} \overline{B_{i}}} \sum_{\substack{j \neq i \\
\omega \models A_{j} \overline{B_{j}}}} \eta_{j} \\
&=-\min _{\omega \models A_{i} \overline{B_{i}}} \sum_{\substack{j \neq i \\
\omega \models A_{j} \overline{B_{j}}}} \eta_{j}
\end{aligned}
$$

for all $i \in\{1, \ldots, n\}$. Thus, the constraint (8) reduces to

$$
\eta_{i}>-\min _{\omega \models A_{i} \overline{B_{i}}} \sum_{\substack{j \neq i \\ \omega \models A_{j} \overline{B_{j}}}} \eta_{j}
$$

for all $i \in\{1, \ldots, n\}$.

The implication " $\Longrightarrow$ " from (14) is obvious since $\mathcal{O}\left(C R^{n-1}(\mathcal{R})\right) \subseteq \mathcal{O}(C R(\mathcal{R}))$. For the proof of the other implication " $\Longleftarrow "$ fix formulas $A, B$ such that

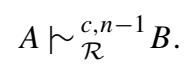

We have to show $A \sim{ }_{\mathcal{R}}^{c} B$. Due to Proposition 1 this requires $\kappa_{\vec{\eta}}(A B)<\kappa_{\vec{\eta}}(A \bar{B})$ for all $\kappa \vec{\eta}$ (defined in (6)) where $\vec{\eta}=\left(\eta_{1}, \ldots, \eta_{n}\right) \in \operatorname{Sol}(C R(\mathcal{R}))$. That is, in turn, equivalent to

$$
\forall \omega^{0} \in \Omega_{A \bar{B}} \exists \omega^{1} \in \Omega_{A B} \text { with } \kappa \vec{\eta}\left(\omega^{1}\right)<\kappa \vec{\eta}\left(\omega^{0}\right) .
$$

Fix any c-representation $\kappa_{\vec{\eta}} \in \mathcal{O}(C R(\mathcal{R}))$ with $\vec{\eta}=\left(\eta_{1}, \ldots, \eta_{n}\right)$ and $\eta_{i} \geq 0, i \in$ $\{1, \ldots, n\}$. Further, fix $\omega^{0} \in \Omega_{A \bar{B}}$. Let us define the set of all indices such that $\eta_{i}>0$ and the corresponding conditional is falsified by $\omega^{0}$ as

$$
J:=\left\{i \in\{1, \ldots, n\} ; \eta_{i}>0 \text { and } \omega^{0} \models A_{i} \overline{B_{i}}\right\} .
$$


Our goal is to construct $\omega^{1} \in \Omega_{A B}$ such that

$$
\begin{aligned}
& \left\{i \in\{1, \ldots, n\} ; \eta_{i}>0 \text { and } \omega^{1} \models A_{i} \overline{B_{i}}\right\} \\
& \subsetneq\left\{i \in\{1, \ldots, n\} ; \eta_{i}>0 \text { and } \omega^{0} \models A_{i} \overline{B_{i}}\right\} .
\end{aligned}
$$

Indeed, assume that (26) is proven. From (26) we get immediately

$$
\kappa \vec{\eta}\left(\omega^{1}\right)=\sum_{\substack{i \in\{1, \ldots, n\} \\ \omega^{1} \models A_{i} \overline{B_{i}}}} \eta_{i}<\sum_{\substack{i \in\{1, \ldots, n\} \\ \omega^{0} \models A_{i} \overline{B_{i}}}} \eta_{i}=\kappa \vec{\eta}\left(\omega^{0}\right)
$$

implying that (24) is fulfilled. Altogether, to finish the proof we have to show the existence of $\omega^{1} \in \Omega_{A B}$ with (26). We distinguish the cases as follows.

Case (i). First, let us consider the case $|J|=n$. Choose arbitrary $\eta_{i}^{\prime} \in\{1, \ldots, n-1\}$ for $i \in\{1, \ldots, n\}$. Since $\eta_{i}^{\prime}>0$ it follows that (22) holds (which is equivalent to (8)) and so $\vec{\eta}^{\prime}$ fulfils (7), (8). Due to Proposition 1 it follows $\kappa \vec{\eta}^{\prime} \in \mathcal{O}\left(C R^{n-1}(\mathcal{R})\right)$. From (23) we get $\omega^{1} \in \Omega_{A B}$ with $\kappa_{\vec{\eta}^{\prime}}\left(\omega^{1}\right)<\kappa_{\eta^{\prime}}\left(\omega^{0}\right)$. Thus $\sum_{\substack{i \in\{1, \ldots, n\} \\ \omega^{1} \models A_{i}, \overline{B_{i}}}} \eta_{i}^{\prime}<\sum_{i=1}^{n} \eta_{i}^{\prime}$. However, this yields

$$
\left\{i \in\{1, \ldots, n\} ; \omega^{1} \models A_{i} \overline{B_{i}}\right\} \subsetneq\{1, \ldots, n\} .
$$

Consequently (26) holds.

Case (ii). Let us consider the case $|J|<n$. We define $\vec{\eta}^{\prime}=\left(\eta_{1}^{\prime}, \ldots, \eta_{n}^{\prime}\right)$ by

$$
\eta_{i}^{\prime}:= \begin{cases}0, & \text { if } i \in\{1, \ldots n\} \text { with } \eta_{i}=0, \\ 1, & \text { if } i \in J, \\ n-1, & \text { otherwise. }\end{cases}
$$

Since $\eta_{i}^{\prime} \geq 0, i \in\{1, \ldots, n\}$ it remains to prove the constraint (8) which we know is equivalent to (22). If $i \in\{1, \ldots, n\}$ such that $\eta_{i}^{\prime}>0$ then obviously (22) holds. Therefore let us consider $i \in\{1, \ldots, n\}$ such that $\eta_{i}^{\prime}=0$. Since $\eta_{i}=0$ we know that (22) holds. Thus

$$
0>-\min _{\omega \models A_{i} \overline{B_{i}}} \sum_{\substack{j \neq i \\ \omega \models A_{j} \overline{B_{j}}}} \eta_{j}
$$

and so $\min _{\omega \models A_{i} \overline{B_{i}}} \sum_{j \neq i} \eta_{j} \in \mathbb{N}^{\infty}$ (i.e. $\neq 0$ ). By (27) we have $\eta_{j}>0$ implies $\eta_{j}^{\prime}>0$ and $\omega \models A_{j} \overline{B_{j}}$

so $\min _{\omega \models A_{i} \overline{B_{i}}} \sum_{\substack{j \neq i \\ \omega \models A_{j} \overline{B_{j}}}} \eta_{j}^{\prime} \in \mathbb{N}^{\infty}$. Consequently (8) is also satisfied for $\eta_{i}^{\prime}=0$. Making use of

Proposition 1 it follows that $\kappa \vec{\eta}^{\prime} \in \mathcal{O}\left(C R^{n-1}(\mathcal{R})\right)$. By (23) we know $A \sim^{\kappa} \vec{\eta}^{\prime} B$. Therefore, there is $\omega^{1} \in \Omega_{A B}$ with $\kappa \vec{\eta}^{\prime}\left(\omega^{1}\right)<\kappa \vec{\eta}^{\prime}\left(\omega^{0}\right)$. Thus

$$
\sum_{\substack{i \in\{1, \ldots, n\} \\ \omega^{1} \models A_{i} \overline{B_{i}}}} \eta_{i}^{\prime}<\sum_{\substack{i \in\{1, \ldots, n\} \\ \omega^{0} \models A_{i} \bar{B}_{i}}} \eta_{i}^{\prime}
$$


By definition of $J$ and $\vec{\eta}^{\prime}$, see (25), (27), it holds for all $i \in\{1, \ldots, n\}$ with $\eta_{i}>0$ and $\omega^{0} \models A_{i} \overline{B_{i}}$ that $\eta_{i}^{\prime}=1$. Therefore $\sum_{\substack{i \in\{1, \ldots, n\} \\ \omega^{0} \models A_{i} \bar{B}_{i}}} \eta_{i}^{\prime}=|J|$. By (28) we get

$$
\sum_{\substack{i \in\{1, \ldots, n\} \\ \omega^{1} \models A_{i} \overline{B_{i}}}} \eta_{i}^{\prime}<|J| \leq n-1 .
$$

We finish the proof of (26) by contradiction. Suppose that (26) is wrong. Due to (28) the two sets in (26) can not be identical. Therefore, there must be an $k \in\{1, \ldots, n\}$ such that $\eta_{k}>0$ and $\omega^{1} \models A_{k} \overline{B_{k}}$ but not $k \in J$. Since $k \notin J$, by (27), there holds $\eta_{k}^{\prime}=n-1$. But then

$$
\sum_{\substack{i \in\{1, \ldots, n\} \\ \omega^{1} \models A_{i} \overline{B_{i}}}} \eta_{i}^{\prime}=n-1+\sum_{\substack{i \neq k \\ \omega^{1} \models A_{i} \overline{B_{i}}}} \eta_{i}^{\prime} \geq n-1,
$$

contradicting (29). Altogether the existence of $\omega^{1}$ with (26) is also proven in the case (ii). The proof is complete.

We close this section by comparing Proposition 7 to the results of [9] for the knowledge base $\mathcal{R}_{n}=\left\{\left(a_{1} \mid T\right), \ldots,\left(a_{n} \mid \top\right)\right\}$ of $n$ conditional facts (c.f. Example 9). Since $\omega=a_{1} \ldots a_{n}$ accepts all conditionals from $\mathcal{R}_{n}$, our more general Proposition 7 yields the same conclusion for $\mathcal{R}_{n}$ as in [9, Proposition 19], but makes no use of the special structure of the conditionals from $\mathcal{R}_{n}$.

\section{Existence of a knowledge base such that $2^{n-1}$ is minimally sufficient and minimally regular}

In [9], the authors state the conjecture that a maximal impact factor of $u=|\mathcal{R}|$ is sufficient for all knowledge bases $\mathcal{R}$; this conjecture was supported by empirical experiments using the reasoning platform InfOCF $[6,9]$. However, in this section, we will construct a knowledge base $\mathcal{R}=\left\{\left(B_{i} \mid A_{i}\right), i=1, \ldots, n\right\}$ where $u=2^{n-1}$ is minimally sufficient and minimally regular for $\mathcal{R}$. Therefore, in general, the exponential lower bound $u=2^{n-1}$ is needed as possible impact factor for solutions of the finite domain constraint satisfaction problem $C R^{u}(\mathcal{R})$ (Definition 5) to fully realize (skeptical) c-inference. The construction of a knowledge base requiring an exponential impact factor is based on the existence of a knowledge base $\mathcal{R}$ such that the solutions of the general CSP $C R(\mathcal{R})$ (Definition 3) and the restricted CSP $C R_{o p}(\mathcal{R})$ (Definition 10) coincide. The proof of this latter result is based on on the observation that every given verification/falsification behavior of conditionals on worlds can be realized by a knowledge base. In this section, we will elaborate all technical details required for the construction of $\mathcal{R}$ and prove its claimed properties.

After having outlined the content of the present section, let us consider $\Omega=\left\{\omega_{i} ; i=\right.$ $1, \ldots, m\}$. For conditionals $\left(B_{j} \mid A_{j}\right)_{j=1, \ldots, n}$ a matrix $\left(m_{i, j}\right)$ with $m_{i, j} \in\{\mathrm{v}, \mathrm{f},-\}$ describing the evaluation according to (1) can be defined by

$$
m_{i, j}=\left[\left[\left(B_{j} \mid A_{j}\right)\right]\right]_{\omega_{i}} .
$$

In (30) $m_{i, j}=\mathrm{v}$ means that $\omega_{i}$ verifies $\left(B_{j} \mid A_{j}\right)$, the meaning of $m_{i, j}=\mathrm{f}$ is that $\omega_{i}$ falsifies $\left(B_{j} \mid A_{j}\right)$ and we write $m_{i, j}=-$ if $\omega_{i} \models \overline{A_{j}}$. 
In the following proposition we tackle the "inverse problem": For a given evaluation matrix $\left(m_{i, j}\right)_{i=1, \ldots, m} ; j=1, \ldots, n$ we construct a (not necessarily consistent) knowledge base $\mathcal{R}=\left\{\left(B_{j} \mid A_{j}\right), j=1, \ldots, n\right\}$ such that the evaluation is just given by (30).

Proposition 8 Let $n, m \in \mathbb{N}$, let $\Sigma=\left\{v_{1}, \ldots, v_{m}\right\}$ be a propositional alphabet and let $\Omega=\left\{\omega_{1}, \ldots, \omega_{2^{m}}\right\}$. For all $i \in\left\{1, \ldots, 2^{m}\right\}$ and $j \in\{1, \ldots, n\}$ let $m_{i, j} \in\{\mathrm{v}, \mathrm{f},-\}$ be given. Then there exists a (not necessarily consistent) knowledge base $\mathcal{R}=\left\{\left(B_{j} \mid A_{j}\right), j=\right.$ $1, \ldots, n\}$ such that the following holds:

$$
\begin{aligned}
& \text { If } m_{i, j}=\mathrm{v} \text { then } \omega_{\mathrm{i}} \models \mathrm{A}_{\mathrm{j}} \mathrm{B}_{\mathrm{j}}, \\
& \text { If } m_{i, j}=\mathrm{f} \text { then } \omega_{\mathrm{i}}=\mathrm{A}_{\mathrm{j}} \overline{\mathrm{B}_{\mathrm{j}}}, \\
& \text { If } m_{i, j}=- \text { then } \omega_{i} \models \overline{A_{j}} .
\end{aligned}
$$

Proof We have to construct $r_{j}=\left(B_{j} \mid A_{j}\right)$ such that $m_{i, j}=\left[\left[\left(B_{i} \mid A_{i}\right)\right]\right]_{\omega_{i}}$. To do so we define the formulas

$$
\begin{aligned}
A_{j}:= & \bigvee_{\left\{\omega_{i} \in \Omega ; m_{i, j} \in\{\mathrm{v}, f\}\right\}} \omega_{i}, \\
B_{j} & :=\bigvee_{\left\{\omega_{i} \in \Omega ; m_{i, j}=\mathrm{v}\right\}} \omega_{i}
\end{aligned}
$$

for all $j \in\{1, \ldots, n\}$. Due to construction we see that (31), (32), (33) hold.

Proposition 8 can be summarized as follows: Every verification/falsification behavior (see Table 4 ), described by an evaluation matrix $\left(m_{i, j}\right)_{i=1, \ldots, m ; j=1, \ldots, n}$, can be generated by a knowledge base $\mathcal{R}=\left\{\left(B_{j} \mid A_{j}\right), j=1, \ldots, n\right\}$.

The following main result shows the existence of knowledge bases such that the solutions of $C R(\mathcal{R})$ and $C R_{o p}(\mathcal{R})$ coincide.

Proposition 9 Let $n \in \mathbb{N}$, let $k \in \mathbb{N}_{0}$ with $n \geq k+1$, and let $s_{m} \in \mathbb{N}, m=0, \ldots, k$, with $\sum_{m=0}^{k} s_{m}=n$. Then there exists a consistent knowledge base $\mathcal{R}=\left\{r_{1}, \ldots, r_{n}\right\}$ with inclusion-maximal partition $\operatorname{OP}(\mathcal{R})=\left(\mathcal{R}_{0}, \ldots, \mathcal{R}_{k}\right)$ with $\left|\mathcal{R}_{m}\right|=s_{m}$ for $m \in\{0, \ldots, k\}$ such that

$$
\operatorname{Sol}(C R(\mathcal{R}))=\operatorname{Sol}\left(C R_{o p}(\mathcal{R})\right)
$$

Proof Let $\Sigma=\left\{v_{1}, \ldots v_{u}\right\}$ be a propositional alphabet and, as usual, we identify the set of all possible worlds with the set of complete conjunctions over $\Sigma$. We choose $u \in \mathbb{N}$ such that $|\Omega|=2^{u} \geq n+k+1$. If $n>1$ then $u=n$ is possible, if $n=1$ we have to choose $u \geq 2$.

Table 4 Verification/falsification behavior described by the matrix $m_{i, j}$

\begin{tabular}{llllll}
\hline worlds & $r_{1}$ & $r_{2}$ & $r_{3}$ & $\ldots$ & $r_{n}$ \\
\hline$\omega_{1}$ & $m_{1,1}$ & $m_{1,2}$ & $m_{1,3}$ & $\ldots$ & $m_{1, n}$ \\
$\omega_{2}$ & $m_{2,1}$ & $m_{2,2}$ & $m_{2,3}$ & $\ldots$ & $m_{2, n}$ \\
$\omega_{3}$ & $m_{3,1}$ & $m_{3,2}$ & $m_{3,3}$ & $\ldots$ & $m_{3, n}$ \\
$\ldots$ & & & & $\ldots$ & $m_{2^{m}, n}$ \\
$\omega_{2^{m}}$ & $m_{2^{m}, 1}$ & $m_{2^{m}, 2}$ & $m_{2^{m}, 3}$ & $\ldots$ & \\
\hline
\end{tabular}


Consider disjoint subsets $\Omega_{+}, \Omega_{-} \subseteq \Omega$ where $\left|\Omega_{+}\right|=k+1$ and $\left|\Omega_{-}\right|=n$. Let us write $\Omega=\Omega_{+} \cup \Omega_{-} \cup \Omega_{\text {rest }}$ where

$$
\begin{aligned}
\Omega_{-} & =\left\{w_{1}^{-}, w_{2}^{-}, \ldots, w_{n}^{-}\right\}, \\
\Omega_{+} & =\left\{w_{1}^{+}, w_{2}^{+}, \ldots, w_{k+1}^{+}\right\}, \\
\Omega_{\text {rest }} & =\Omega \backslash\left(\Omega_{-} \cup \Omega_{+}\right) .
\end{aligned}
$$

Looking at Proposition 8 there exists a knowledge base

$$
\mathcal{R}=\left\{r_{j}^{(m)} \mid m \in\{0, \ldots, k\} \text { and } j \in\left\{1, \ldots, s_{m}\right\}\right\}
$$

fulfilling the verification/falsification behavior described in Table 5 .

Looking at this table we see that the ordered partition $\left(\mathcal{R}_{0}, \ldots \mathcal{R}_{k}\right)$ where

$$
\mathcal{R}_{m}=\left\{r_{l}^{(m)} \mid l=1, \ldots, s_{m}\right\}
$$

is in fact the inclusion-maximal partition to $\mathcal{R}$, especially $\mathcal{R}$ is consistent. Let us write $\mathcal{R}=\left\{r_{1}, \ldots, r_{n}\right\}$. The following holds:

- For every $m \in\{0, \ldots, k\}$ we have that $\omega_{m+1}^{+}$falsifies all conditionals from $\bigcup_{l=0}^{m-1} \mathcal{R}_{l}$, verifies every conditional from $\mathcal{R}_{m}$ and is not applicable on all conditionals from $\bigcup_{l=m+1}^{k} \mathcal{R}_{l}$.

- For $i \in\{1, \ldots, n\}$ we have that $\omega_{i}^{-}$falsifies $r_{i}$ and is not applicable on all other conditionals.

Due to construction

$$
\min _{\omega \models A_{i} \overline{B_{i}}} \sum_{\substack{j \neq i \\ \omega \models A_{j} \overline{B_{j}}}} \eta_{j}=0
$$

Table 5 Verification/falsification behavior to obtain $\mathcal{R}$ as in Proposition 9

\begin{tabular}{llllllllllllll}
\hline worlds & $r_{1}^{(0)}$ & $\ldots$ & $r_{s_{0}}^{(0)}$ & $r_{1}^{(1)}$ & $\ldots$ & $r_{s_{1}}^{(1)}$ & $r_{1}^{(2)}$ & $\ldots$ & $r_{s_{2}}^{(2)}$ & $\ldots$ & $r_{1}^{(k)}$ & $\ldots$ & $r_{s_{k}}^{(k)}$ \\
\hline$\omega_{1}^{+}$ & $\mathrm{v}$ & $\ldots$ & $\mathrm{v}$ & - & $\ldots$ & - & - & $\ldots$ & - & $\ldots$ & - & $\ldots$ & - \\
$\omega_{2}^{+}$ & $\mathrm{f}$ & $\ldots$ & $\mathrm{f}$ & $\mathrm{v}$ & $\ldots$ & $\mathrm{v}$ & - & $\ldots$ & - & $\ldots$ & - & $\ldots$ & - \\
$\omega_{3}^{+}$ & $\mathrm{f}$ & $\ldots$ & $\mathrm{f}$ & $\mathrm{f}$ & $\ldots$ & $\mathrm{f}$ & $\mathrm{v}$ & $\ldots$ & $\mathrm{v}$ & $\ldots$ & - & $\ldots$ & - \\
$\ldots$ & & & & & & & & & & & & & \\
$\omega_{k+1}^{+}$ & $\mathrm{f}$ & $\ldots$ & $\mathrm{f}$ & $\mathrm{f}$ & $\ldots$ & $\mathrm{f}$ & $\mathrm{f}$ & $\ldots$ & $\mathrm{f}$ & $\ldots$ & $\mathrm{v}$ & $\ldots$ & $\mathrm{v}$ \\
$\omega_{1}^{-}$ & $\mathrm{f}$ & $\ldots$ & - & - & $\ldots$ & - & - & $\ldots$ & - & $\ldots$ & - & $\ldots$ & - \\
$\omega_{s_{0}}^{-}$ & - & $\ldots$ & $\mathrm{f}$ & - & $\ldots$ & - & - & $\ldots$ & - & $\ldots$ & - & $\ldots$ & - \\
$\omega_{s_{0}+1}^{-}$ & - & $\ldots$ & - & $\mathrm{f}$ & $\ldots$ & - & - & $\ldots$ & - & $\ldots$ & - & $\ldots$ & - \\
$\ldots$ & & & & & & & & & & & & & $\ldots$ \\
$\omega_{n}^{-}$ & - & $\ldots$ & - & - & $\ldots$ & - & - & $\ldots$ & - & $\ldots$ & - & $\ldots$ \\
all other worlds & - & $\ldots$ & - & - & $\ldots$ & - & - & $\ldots$ & - & $\ldots$ & - & $\ldots$ & - \\
\hline
\end{tabular}


for all $i \in\{1, \ldots, n\}$. Consider $i \in\{1, \ldots, n\}$ and let $r_{i}=r_{l}^{(m)}$ with $m=m(i) \in$ $\{0, \ldots, k\}$ and $l=l(i) \in\left\{1, \ldots, s_{m}\right\}$. Since $\omega_{m+1}^{+}$is the only world verifying $r_{i}$ we get using the properties of $\omega_{m+1}^{+}$that

$$
\min _{\omega \models A_{i} B_{i}} \sum_{\substack{j \neq i \\ \omega \models A_{j} \overline{B_{j}}}} \eta_{j}=\sum_{\substack{j \neq i \\ \omega_{m+1}^{+} \models A_{j} \overline{B_{j}}}} \eta_{j}=\sum_{\substack{j \in\{1, \ldots, n\} \\ r_{j} \in \bigcup_{l=0}^{m-1} \mathcal{R}_{l}}} \eta_{j} .
$$

Inserting (37), (38) in (8) yields

$$
\eta_{i}>\sum_{\substack{j \in\{1, \ldots, n\} \\ r_{j} \in \bigcup_{l=0}^{m-1} \mathcal{R}_{l}}} \eta_{j}
$$

Further if (39) holds then (7) is automatically fulfilled. Altogether $C R(\mathcal{R})$ reduces to the conjunction of (39) for all $i \in\{1, \ldots, n\}$. Since (39) is exactly the constraint defining $C R_{o p}(\mathcal{R})$ we obtain (36), finishing the proof.

In [6], three different notions of minimality for the solution vectors in $\operatorname{Sol}(C R(\mathcal{R}))$ are introduced. If $\mathcal{R}$ is consistent, then minimal solutions for all three notions of minimality exist, but none of them is necessarily unique.

Definition 11 (absolute minimal solution) Let $\mathcal{R}$ be a knowledge base and let $\vec{\eta}=$ $\left(\eta_{1}, \ldots, \eta_{n}\right)$ be a solution of $C R(\mathcal{R})$. Then $\vec{\eta}$ is called absolute minimal solution of $C R(\mathcal{R})$ if for every solution $\left(\eta_{1}^{\prime}, \ldots, \eta_{n}^{\prime}\right)$ of $C R(\mathcal{R})$ we have $\eta_{i} \leq \eta_{i}^{\prime}$ for all $i \in\{1, \ldots, n\}$.

Note that even if $\mathcal{R}$ is consistent, an absolute minimal solution of $C R(\mathcal{R})$ may not exist. On the other hand, it follows immediately from the definition that an absolute minimal solution of $C R(\mathcal{R})$, if such a solution exists, is uniquely determined. Furthermore, an absolute minimal solution solution of $C R(\mathcal{R})$ is also cw-minimal, ind-minimal and sum-minimal in the sense of [6].

Using Proposition 9, we can prove the existence of a knowledge base $\mathcal{R}$ such that the absolute minimal solution of $C R(\mathcal{R})$ exists and that this absolute minimal solution of $C R(\mathcal{R})$ has an impact that is exponential in the number of conditionals in $\mathcal{R}$.

Corollary 1 There exists a consistent knowledge base $\mathcal{R}=\left\{\left(B_{i} \mid A_{i}\right), i=1, \ldots, n\right\}$, such that

$$
\operatorname{Sol}(C R(\mathcal{R}))=\left\{\left(\eta_{1}, \ldots, \eta_{n}\right) \in \mathbb{N}_{0}^{n} \mid \eta_{i}>\sum_{j=1}^{i-1} \eta_{j}\right\} .
$$

The $\operatorname{CSP} C R(\mathcal{R})$ has the absolute minimal solution

$$
\vec{\eta}=\left(1,2,4,8, \ldots, 2^{n-1}\right) .
$$

Proof Define $k:=n-1$ and $s_{m}:=1$ for $m \in\{0, \ldots, k\}$. From Proposition 9 we obtain a consistent knowledge base $\mathcal{R}$ with inclusion-maximal partition $\left(\mathcal{R}_{0}, \ldots, \mathcal{R}_{k}\right)$ fulfilling $\left|\mathcal{R}_{m}\right|=s_{m}$ for all $m \in\{0, \ldots, k\}$. We write $\mathcal{R}=\left\{r_{1}, \ldots, r_{n}\right\}$ such that $\mathcal{R}_{m}=\left\{r_{m+1}\right\}$ for all $m \in\{0, \ldots, k\}$. We have to show (40), (41). 
Fix $i \in\{1, \ldots, n\}$. Then $r_{i} \in \mathcal{R}_{i-1}$ and consequently we obtain from (39) that $C R(\mathcal{R})$ is given by the conjunction of

$$
\eta_{i}>\sum_{\substack{j \in\{1, \ldots, n\} \\ r_{j} \in \bigcup_{l=0}^{m-1} \mathcal{R}_{l}}} \eta_{j}=\sum_{\substack{j \in\{1, \ldots, n\} \\ r_{j} \in \bigcup_{l=0}^{i-1} \mathcal{R}_{l}}} \eta_{j}=\sum_{j=1}^{i-1} \eta_{j}
$$

for all $i \in\{1, \ldots, n\}$. Consequently (40) holds.

Looking at (42) it follows that a solution to $C R(\mathcal{R})$ is given by $\vec{\eta}=\left(\eta_{1}, \ldots, \eta_{n}\right)$ where

$$
\eta_{i}=1+\sum_{j=1}^{i-1} \eta_{j}
$$

for all $i \in\{1, \ldots, n\}$. Moreover, due to the simple structure of (42), we immediately see that $\vec{\eta}=\left(\eta_{1}, \ldots, \eta_{n}\right)$ as in (43) is indeed the absolute minimal solution of $C R(\mathcal{R})$. The proof of the representation (41) is by induction. For $i=1$ we have $\eta_{1}=1=2^{1-1}$. The proof of the inductive step follows from

$$
\eta_{i}=1+\sum_{j=1}^{i-1} \eta_{j}=1+\sum_{j=0}^{i-2} 2^{j}=1+\frac{1-2^{i-1}}{1-2}=2^{i-1} .
$$

Consequently (41) holds.

Let us explain and clarify the construction of a possible realization of a knowledge base $\mathcal{R}=\left\{r_{i}=\left(B_{i} \mid A_{i}\right), i=1, \ldots, n\right\}$ whose existence is stated in Corollary 1. In Proposition 10 we will show that for such a knowledge base $2^{n-1}$ is minimally sufficient and minimally regular. Let $\Sigma=\left\{v_{1}, \ldots v_{u}\right\}$ be a propositional alphabet where $u \in \mathbb{N}$ is chosen such that $|\Omega|=2^{u} \geq 2 n$. Consider disjoint subsets $\Omega_{+}, \Omega_{-} \subseteq \Omega$ where $\left|\Omega_{+}\right|=n$ and $\left|\Omega_{-}\right|=n$. Let us write $\Omega=\Omega_{+} \cup \Omega_{-} \cup \Omega_{\text {rest }}$ where

$$
\begin{aligned}
\Omega_{-} & =\left\{\omega_{1}^{-}, \omega_{2}^{-}, \ldots, \omega_{n}^{-}\right\}, \\
\Omega_{+} & =\left\{\omega_{1}^{+}, \omega_{2}^{+}, \ldots, \omega_{n}^{+}\right\}, \\
\Omega_{\text {rest }} & :=\Omega \backslash\left(\Omega_{-} \cup \Omega_{+}\right) .
\end{aligned}
$$

An inspection of the proof of Proposition 9 and Corollary 1 shows that our goal is to construct $\mathcal{R}$ with the verification/falsification behavior described by Table 6 .

By (34), (35) in the proof of Proposition 8 it follows that we can choose the knowledge base $\mathcal{R}=\left\{r_{i}=\left(B_{i} \mid A_{i}\right), i=1, \ldots, n\right\}$ in the following way

$$
\begin{aligned}
A_{i} & :=\bigvee_{\left\{\omega \in \Omega ;\left[\left[r_{i}\right]\right]_{\omega} \in\{\mathrm{f}, \mathrm{v}\}\right\}} \omega=\omega_{i}^{-} \vee \omega_{i}^{+} \vee \ldots \vee \omega_{n}^{+}, \\
B_{i} & \left.:=\bigvee_{\left\{\omega \in \Omega ;\left[\left[r_{i}\right]\right]_{\omega}=\mathrm{v}\right.}\right\} \omega=\omega_{i}^{+}
\end{aligned}
$$

for all $i \in\{1, \ldots, n\}$. Consequently we obtain

$$
r_{i}=\left(\omega_{i}^{+} \mid \omega_{i}^{-} \vee \omega_{i}^{+} \vee \ldots \vee \omega_{n}^{+}\right) .
$$

The following remarkable lemma states that for every knowledge base $\mathcal{R}$ with $C R(\mathcal{R})$ given by (40) all c-representations of $\mathcal{R}$ are inferentially equivalent.

Lemma 1 Let $\mathcal{R}=\left\{\left(B_{i} \mid A_{i}\right), i=1, \ldots, n\right\}$ denote a consistent knowledge base fulfilling (40). Then every c-representation $\kappa$ for $\mathcal{R}$ is inferentially equivalent to $\kappa \vec{\eta}$ (defined 
Table 6 Verification/falsification behavior to obtain $\mathcal{R}$ as in Corollary 1

\begin{tabular}{|c|c|c|c|c|c|c|}
\hline worlds & $r_{1}$ & $r_{2}$ & $r_{3}$ & $\ldots$ & $r_{n-1}$ & $r_{n}$ \\
\hline$\omega_{1}^{+}$ & v & - & - & $\ldots$ & - & - \\
\hline$\omega_{2}^{+}$ & $f$ & v & - & $\ldots$ & - & - \\
\hline$\omega_{3}^{+}$ & $f$ & $f$ & v & $\ldots$ & - & - \\
\hline \multicolumn{7}{|l|}{$\ldots$} \\
\hline$\omega_{n-1}^{+}$ & $f$ & $f$ & $f$ & $\ldots$ & v & - \\
\hline$\omega_{n}^{+}$ & $f$ & $f$ & $f$ & $\ldots$ & $f$ & v \\
\hline$\omega_{1}^{-}$ & $f$ & - & - & $\ldots$ & - & - \\
\hline$\omega_{2}^{-}$ & - & $f$ & - & $\ldots$ & - & - \\
\hline$\omega_{3}^{-}$ & - & - & $f$ & $\cdots$ & - & - \\
\hline \multicolumn{7}{|l|}{$\ldots$} \\
\hline$\omega_{n-1}^{-}$ & - & - & - & $\ldots$ & $f$ & - \\
\hline$\omega_{n}^{-}$ & - & - & - & $\ldots$ & - & $f$ \\
\hline all other worlds & - & - & - & $\ldots$ & - & - \\
\hline
\end{tabular}

in (6)) where $\vec{\eta}$ is the absolute minimal solution solution to $C R(\mathcal{R})$ given by $\vec{\eta}=$ $\left(1,2,4,8, \ldots, 2^{n-1}\right)$.

Proof Let $\kappa$ be an arbitrary c-representation for $\mathcal{R}$ and let $\omega_{1}, \omega_{2} \in \Omega$. Let us define

$$
\begin{aligned}
& J_{1}:=\left\{i \in\{1, \ldots, n\} ; \omega_{1} \models A_{i} \overline{B_{i}}\right\}, \\
& J_{2}:=\left\{i \in\{1, \ldots, n\} ; \omega_{2} \models A_{i} \overline{B_{i}}\right\} .
\end{aligned}
$$

Let us write $\max M=0$ if $M \subseteq\{1, \ldots, n\}$ with $M=\varnothing$.

Assertion. We have

$$
\begin{aligned}
& \kappa\left(\omega_{1}\right)>\kappa\left(\omega_{2}\right) \Longleftrightarrow \max \left(J_{1} \backslash J_{2}\right)>\max \left(J_{2} \backslash J_{1}\right), \\
& \kappa\left(\omega_{1}\right)=\kappa\left(\omega_{2}\right) \Longleftrightarrow J_{1}=J_{2} .
\end{aligned}
$$

Proof of the assertion. By Proposition 1 there is $\vec{\eta}^{\prime}=\left(\eta_{1}^{\prime}, \ldots, \eta_{n}^{\prime}\right) \in \operatorname{Sol}(C R(\mathcal{R}))$ such that $\kappa=\kappa \vec{\eta}^{\prime}$. Since (46) implies (47) it remains to show (46). We obtain

$$
\kappa\left(\omega_{1}\right)-\kappa\left(\omega_{2}\right)=\sum_{j \in J_{1}} \eta_{j}-\sum_{j \in J_{2}} \eta_{j}^{\prime}=\sum_{j \in J_{1} \backslash J_{2}} \eta_{j}^{\prime}-\sum_{j \in J_{2} \backslash J_{1}} \eta_{j}^{\prime} .
$$

Define $q_{1}:=\max J_{1}$ and $q_{2}:=\max J_{2}$. Assume $\max \left(J_{1} \backslash J_{2}\right)>\max \left(J_{2} \backslash J_{1}\right)$. Then $q_{1}>q_{2}$ and it follows

$$
\begin{aligned}
\kappa\left(\omega_{1}\right)-\kappa\left(\omega_{2}\right) & =\sum_{j \in J_{1} \backslash J_{2}} \eta_{j}-\sum_{j \in J_{2} \backslash J_{1}} \eta_{j} \\
& \geq \eta_{q_{1}}-\sum_{j \in\left\{1, \ldots, q_{1}-1\right\}} \eta_{j}>0
\end{aligned}
$$

due to the structure of $\operatorname{Sol}(C R(\mathcal{R}))$. On the other hand if

$$
\sum_{j \in J_{1} \backslash J_{2}} \eta_{j}^{\prime}-\sum_{j \in J_{2} \backslash J_{1}} \eta_{j}^{\prime}=\kappa\left(\omega_{1}\right)-\kappa\left(\omega_{2}\right)>0
$$


it follows due to the structure of $\operatorname{Sol}(C R(\mathcal{R}))$ that $\max \left(J_{1} \backslash J_{2}\right)>\max \left(J_{2} \backslash J_{1}\right)$. The proof of the assertion is complete.

Let $\kappa, \kappa^{\prime} \in \mathcal{O}(C R(\mathcal{R}))$ be c-representations. Making use of the proven assertion we see that

$$
\kappa\left(\omega_{1}\right) \leq \kappa\left(\omega_{2}\right) \quad \Longleftrightarrow \kappa^{\prime}\left(\omega_{1}\right) \leq \kappa^{\prime}\left(\omega_{2}\right)
$$

for all $\omega_{1}, \omega_{2} \in \Omega$. Due to Proposition 2 we get that $\kappa$ and $\kappa^{\prime}$ are inferentially equivalent. The claim follows.

Example 10 By employing Lemma 1, we can investigate further properties of a possible realization of a knowledge base $\mathcal{R}=\left\{r_{i}=\left(B_{i} \mid A_{i}\right), i=1, \ldots, n\right\}$ whose existence is stated in Corollary 1. Let us consider a knowledge base $\mathcal{R}$ with verification/falsification behavior as in Table 6, see formulas (44), (45). We get for the system $\mathrm{Z}$ ranking function $\kappa^{Z}$

$$
\begin{aligned}
& \kappa^{Z}\left(\omega_{i}^{+}\right)=i-1, \\
& \kappa^{Z}\left(\omega_{i}^{-}\right)=i, \\
& \kappa^{Z}(\omega)=0 \text { for all other worlds } .
\end{aligned}
$$

Consider $\kappa \vec{\eta}$ with $\vec{\eta}=\left(1,2,4,8, \ldots, 2^{n-1}\right)$. Then

$$
\begin{aligned}
& \kappa \vec{\eta}\left(\omega_{i}^{+}\right)=\sum_{j=0}^{i-2} 2^{j}=\frac{1-2^{i-1}}{1-2}=2^{i-1}-1, \\
& \kappa \vec{\eta}\left(\omega_{i}^{-}\right)=2^{i-1}, \\
& \kappa \vec{\eta}(\omega)=0 \text { for all other worlds } .
\end{aligned}
$$

Looking at (48), (49) if follows from Proposition 2 that $\kappa^{z}$ and $\kappa_{\vec{\eta}}$ are inferentially equivalent, written as a formula $\kappa^{z} \equiv_{\uparrow} \kappa \vec{\eta}$. Moreover, due to Lemma 1, it follows that system $\mathrm{Z}$ inference and skeptical c-inference coincide for a knowledge base $\mathcal{R}$ with verification/falsification behavior as in Table 6 .

Now we have all ingredients at hand to prove the main result of this section.

Proposition 10 For every $n \in \mathbb{N}$ there exists a consistent knowledge base $\mathcal{R}=$ $\left\{\left(B_{i} \mid A_{i}\right), i=1, \ldots, n\right\}$ such that $2^{n-1}$ is minimally sufficient and minimally regular.

Proof Let $\mathcal{R}$ be the knowledge base whose existence is proven in Corollary 1. Due to Lemma 1, we know that $C R^{2^{n-1}}(\mathcal{R})$ is regular and, see Proposition 3, also sufficient. By (41) we have $\vec{\eta}=\left(1,2,4,8, \ldots, 2^{n-1}\right)$ for the absolute minimal solution of $\mathcal{R}$. Since $\eta_{n}=2^{n-1}$, by the definition of an absolute minimal solution, $\left.\operatorname{Sol}\left(C R^{l}(\mathcal{R})\right)\right)=\varnothing$ if $l<2^{n-1}$. For a consistent knowledge base a regular $C R^{l}(\mathcal{R})$ necessarily has a non empty set of solutions $\left.\operatorname{Sol}\left(C R^{l}(\mathcal{R})\right)\right)$. Therefore, $C R^{l}(\mathcal{R})$ is not regular and, by Proposition 3 , also not sufficient for $l<2^{n-1}$. Altogether $2^{n-1}$ is minimally sufficient and minimally regular for $\mathcal{R}$.

The following example illustrates the findings of Proposition 10.

Example 11 In this example (see Proposition 10 for $n=5$ ) we want to present an explicit knowledge base $\mathcal{R}=\left\{r_{i}=\left(B_{i} \mid A_{i}\right), i=1, \ldots, 5\right\}$ with $n=5$ conditionals such that 
$2^{4}=16$ is minimally sufficient and minimally regular for $\mathcal{R}$. Looking at the proof of Proposition 10 our goal is to construct $\mathcal{R}$ such that $C R(\mathcal{R})$ is given by:

$$
\begin{aligned}
& \eta_{1}>0 \\
& \eta_{2}>\eta_{1} \\
& \eta_{3}>\eta_{1}+\eta_{2} \\
& \eta_{4}>\eta_{1}+\eta_{2}+\eta_{3} \\
& \eta_{5}>\eta_{1}+\eta_{2}+\eta_{3}+\eta_{4}
\end{aligned}
$$

An inspection of the proof of Corollary 1 yields that $\mathcal{R}$ can be constructed having the verification/falsification behavior as shown in Table 7.

Due to (34), (35) we finally arrive at the following concrete knowledge base $\mathcal{R}=$ $\left\{r_{1}, \ldots, r_{5}\right\}$ :

$$
\begin{aligned}
& r_{1}=(\bar{a} b c d e \mid \bar{a} b c d e \vee a \bar{b} \bar{c} \bar{d} \bar{e} \vee a \bar{b} c d e \\
& \vee a b \bar{c} d e \vee a b c \bar{d} e \vee a b c d \bar{e}) \text {, } \\
& r_{2}=(a \bar{b} c d e \mid a \bar{b} c d e \vee \bar{a} b \bar{c} \bar{d} \bar{e} \vee a b \bar{c} d e \\
& \vee a b c \bar{d} e \vee a b c d \bar{e}) \text {, } \\
& r_{3}=(a b \bar{c} d e \mid a b \bar{c} d e \vee \bar{a} \bar{b} c \bar{d} \bar{e} \\
& \vee a b c \bar{d} e \vee a b c d \bar{e}) \text {, } \\
& r_{4}=(a b c \bar{d} e \mid a b c \bar{d} e \vee \bar{a} \bar{b} \bar{c} d \bar{e} \vee a b c d \bar{e}), \\
& r_{5}=(a b c d \bar{e} \mid a b c d \bar{e} \vee \bar{a} \bar{b} \bar{c} \bar{d} e) .
\end{aligned}
$$

\section{Preferred structure on worlds}

Aiming at developing a nonmonotonic inference relation combining the advantages of system $\mathrm{Z}$ and skeptical c-inference, we first introduce the new notion of preferred structure on worlds. The definition of this relation with respect to a knowledge base $\mathcal{R}$ takes into account both the tolerance information expressed by the inclusion maximal partition of $\mathcal{R}$ and the structural information which set of conditionals is falsified by a world.

Table 7 Verification/falsification behavior of $\mathcal{R}$ constructed in Example 11

\begin{tabular}{llllll}
\hline worlds & $r_{1}$ & $r_{2}$ & $r_{3}$ & $r_{4}$ & $r_{5}$ \\
\hline $\bar{a} b c d e$ & $\mathrm{v}$ & - & - & - & - \\
$a \bar{b} c d e$ & $\mathrm{f}$ & $\mathrm{v}$ & - & - & - \\
$a b \bar{c} d e$ & $\mathrm{f}$ & $\mathrm{f}$ & $\mathrm{v}$ & - & - \\
$a b c \bar{d} e$ & $\mathrm{f}$ & $\mathrm{f}$ & $\mathrm{f}$ & $\mathrm{v}$ & - \\
$a b c d \bar{e}$ & $\mathrm{f}$ & $\mathrm{f}$ & $\mathrm{f}$ & $\mathrm{f}$ & $\mathrm{v}$ \\
$a \bar{b} \bar{c} \bar{d} \bar{e}$ & $\mathrm{f}$ & - & - & - & - \\
$\bar{a} b \bar{c} \bar{d} \bar{e}$ & - & $\mathrm{f}$ & - & - & - \\
$\bar{a} \bar{b} c \bar{d} \bar{e}$ & - & - & $\mathrm{f}$ & - & - \\
$\bar{a} \bar{b} \bar{c} d \bar{e}$ & - & - & - & $\mathrm{f}$ & - \\
$\bar{a} \bar{b} \bar{c} \bar{d} e$ & - & - & - & - & $\mathrm{f}$ \\
all other worlds & - & - & - & - & - \\
\hline
\end{tabular}


Definition $12\left(\xi^{j}, \xi\right.$, preferred structure $<_{\mathcal{R}}^{\mathrm{w}}$ on worlds) Consider a consistent knowledge base $\mathcal{R}=\left\{r_{i}=\left(B_{i} \mid A_{i}\right) \mid i \in\{1, \ldots, n\}\right\}$ with $O P(\mathcal{R})=\left(\mathcal{R}_{0}, \ldots, \mathcal{R}_{k}\right)$. For $j \in\{0, \ldots, k\}, \xi^{j}$ and $\xi$ are the functions mapping worlds to the set of falsified conditionals from the tolerance partition $\mathcal{R}_{j}$ and from $\mathcal{R}$, respectively, given by

$$
\begin{gathered}
\xi^{j}(\omega):=\left\{r_{i} \in \mathcal{R}_{j} \mid \omega \models A_{i} \overline{B_{i}}\right\}, \\
\xi(\omega):=\left\{r_{i} \in \mathcal{R} \mid \omega \models A_{i} \overline{B_{i}}\right\} .
\end{gathered}
$$

The preferred structure on worlds is given by the binary relation $<_{\mathcal{R}}^{\mathrm{W}} \subseteq \Omega \times \Omega$ defined by, for any $\omega, \omega^{\prime} \in \Omega$,

$$
\begin{aligned}
& \omega<_{\mathcal{R}}^{\mathrm{w}} \omega^{\prime} \quad \text { iff } \quad \text { there exists } m \in\{0, \ldots, k\} \text { such that } \\
& \xi^{i}(\omega)=\xi^{i}\left(\omega^{\prime}\right) \quad \forall i \in\{m+1, \ldots, k\}, \text { and } \\
& \xi^{m}(\omega) \varsubsetneqq \xi^{m}\left(\omega^{\prime}\right) \text {. }
\end{aligned}
$$

Thus, $\omega<_{\mathcal{R}}^{\mathrm{w}} \omega^{\prime}$ if and only if $\omega$ falsifies strictly less conditionals than $\omega^{\prime}$ in the partition with the biggest index $m$ in $O P(\mathcal{R})$ where the conditionals falsified by $\omega$ and $\omega^{\prime}$ differ. The preferred structure on worlds will be the basis for defining a new inference relation induced by $\mathcal{R}$. Before formally defining this new inference relation and elaborating its properties, we proceed by illustrating the preferred structure on worlds for a knowledge base $\mathcal{R}$, relating it to c-representations of $\mathcal{R}$, and proving a set of its properties that will be useful for investigating the characteristics and properties of the resulting inference relation.

Example $12\left(<\mathrm{\mathcal {R }}_{\text {bird }}^{\mathrm{w}}\right)$ Let us determine the preferred structure on worlds $<_{\mathcal{R}_{\text {bird }}}^{\mathrm{w}}$ for the knowledge base $\mathcal{R}_{\text {bird }}$ from Example 1 . Its verification/falsification behavior is shown in the left-hand side of Table 2 and is repeated for the reader's convenience in Table 8 , along with the system $\mathrm{Z}$ ranking function $\kappa^{Z}$ for $\mathcal{R}_{\text {bird }}$ which was already given in Table 1 .

We know that the inclusion-maximal partition $O P\left(\mathcal{R}_{\text {bird }}\right)=\left(\mathcal{R}_{0}, \mathcal{R}_{1}\right)$ is given by

$$
\begin{aligned}
& \mathcal{R}_{0}=\left\{r_{1}=(f \mid b)\right\}, \\
& \mathcal{R}_{1}=\left\{r_{2}=(\bar{f} \mid p), r_{3}=(\bar{f} \mid b p), r_{4}=(b \mid p)\right\} .
\end{aligned}
$$

Figure 2 shows the preferred structure on worlds $<_{\mathcal{R}_{\text {bird }}}^{\mathrm{w}}$ for the knowledge base $\mathcal{R}_{\text {bird }}$. An edge $\omega \rightarrow \omega^{\prime}$ between two worlds indicates that $\omega<_{\mathcal{R}_{\text {bird }}}^{\mathrm{W}} \omega^{\prime}$. The full relation $<_{\mathcal{R}_{\text {bird }}}^{\mathrm{W}}$ is obtained from the transitive closure of $\rightarrow$ in Fig. 2.

We proceed with a useful lemma.

Table 8 Verification/falsification behavior of the knowledge base $\mathcal{R}_{\text {bird }}$; (v) indicates verification, (f) falsification, and (-) non-applicability. The OCF $\kappa^{Z}$ is the ranking function obtained from $\mathcal{R}_{\text {bird }}$ using system Z

\begin{tabular}{lllllllll}
\hline$\omega$ & $p b f$ & $p b \bar{f}$ & $p \bar{b} f$ & $p \bar{b} \bar{f}$ & $\bar{p} b f$ & $\bar{p} b \bar{f}$ & $\bar{p} \bar{b} f$ & $\bar{p} \bar{b} \bar{f}$ \\
\hline$r_{1}=(f \mid b)$ & $\vee$ & $\mathrm{f}$ & - & - & $\vee$ & $\mathrm{f}$ & - & - \\
$r_{2}=(\bar{f} \mid p)$ & $\mathrm{f}$ & $\mathrm{v}$ & $\mathrm{f}$ & $\mathrm{v}$ & - & - & - & - \\
$r_{3}=(\bar{f} \mid p b)$ & $\mathrm{f}$ & $\mathrm{v}$ & - & - & - & - & - & - \\
$r_{4}=(b \mid p)$ & $\mathrm{v}$ & $\mathrm{v}$ & $\mathrm{f}$ & $\mathrm{f}$ & - & - & - & - \\
$\kappa^{Z}(\omega)$ & 2 & 1 & 2 & 2 & 0 & 1 & 0 & 0 \\
\hline
\end{tabular}




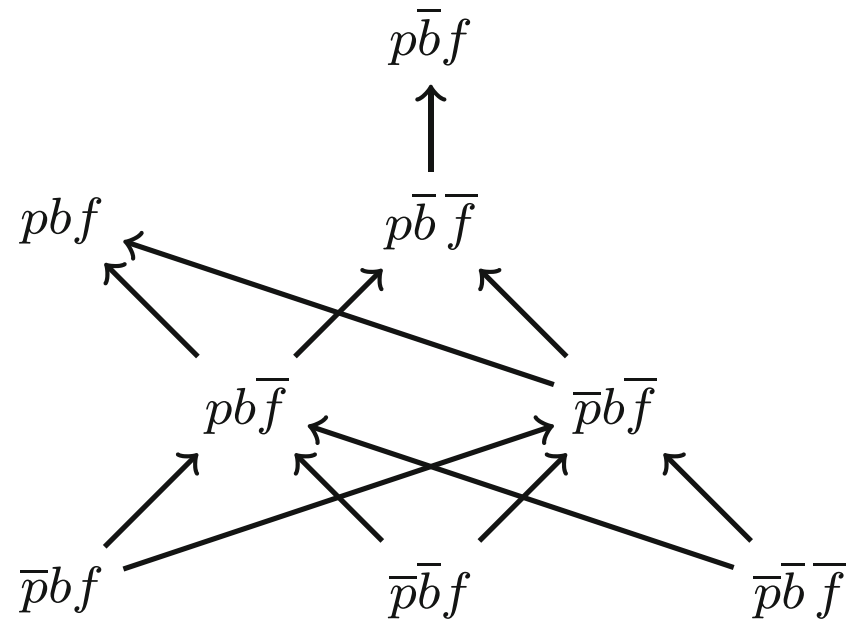

Fig. 2 The preferred structure relation $<_{\mathcal{R}_{\text {bird }}}^{\mathrm{w}}$ on worlds for the knowledge base $\mathcal{R}_{\text {bird }}$

Lemma 2 Let $\mathcal{R}$ be a knowledge base and let $\xi^{i}, \xi$ as in (50) and (51). Consider $\omega, \omega^{\prime} \in$ $\Omega$. Then

$$
\omega \nless_{\mathcal{R}}^{\mathrm{W}} \omega^{\prime}
$$

if and only if $\xi(\omega)=\xi\left(\omega^{\prime}\right)$ or there exists $m \in\{0, \ldots, k\}$ such that

$$
\begin{aligned}
& \xi^{i}(\omega)=\xi^{i}\left(\omega^{\prime}\right) \quad \forall i \in\{m+1, \ldots, k\}, \\
& \xi^{m}(\omega) \nsubseteq \xi^{m}\left(\omega^{\prime}\right) .
\end{aligned}
$$

Proof Consider $\omega, \omega^{\prime} \in \Omega$. Then $\xi(\omega)=\xi\left(\omega^{\prime}\right)$, implying $\omega \nless_{\mathcal{R}}^{\mathrm{w}} \omega^{\prime}$, or there exists a maximal $m \in\{0, \ldots, k\}$ satisfying

$$
\xi^{m}(\omega) \neq \xi^{m}\left(\omega^{\prime}\right) .
$$

Thus

$$
\xi^{i}(\omega)=\xi^{i}\left(\omega^{\prime}\right) \quad \forall i \in\{m+1, \ldots, k\} .
$$

The following two cases can occur:

- $\xi^{m}(\omega) \varsubsetneqq \xi^{m}\left(\omega^{\prime}\right)$. Consequently $\omega<_{\mathcal{R}}^{\mathrm{W}} \omega^{\prime}$.

- $\xi^{m}(\omega) \nsubseteq \xi^{m}\left(\omega^{\prime}\right)$. Consequently $\omega \nless_{\mathcal{R}}^{\mathrm{w}} \omega^{\prime}$.

Thus, in both cases the claim holds.

Example 12 illustrates that $<_{\mathcal{R}}^{\mathrm{w}}$ bird is a strict partial order. The next result states that $<_{\mathcal{R}}^{\mathrm{w}}$ is a strict partial order for every knowledge base $\mathcal{R}$.

Lemma 3 The relation $<_{\mathcal{R}}^{\mathrm{w}}$ is irreflexive, antisymmetric and transitive, meaning that $<_{\mathcal{R}}^{\mathrm{w}}$ is a strict partial order.

Proof Condition (52) immediately yields that $<_{\mathcal{R}}^{\mathrm{w}}$ is irreflexive and antisymmetric. It remains to show that $<_{\mathcal{R}}^{\mathrm{W}}$ is transitive. Define $a:=\max \left\{i \in\{0, \ldots, k\} \mid \xi^{i}\left(\omega_{1}\right) \neq \xi^{i}\left(\omega_{2}\right)\right\}$ and $b:=\max \left\{i \in\{0, \ldots, k\} \mid \xi^{i}\left(\omega_{2}\right) \neq \xi^{i}\left(\omega_{3}\right)\right\}$. Then $\omega_{1}<{ }_{\mathcal{R}}^{\mathrm{w}} \omega_{2}$ and $\omega_{2}<{ }_{\mathcal{R}}^{\mathrm{w}} \omega_{3}$ is equivalent to $\xi^{a}\left(\omega_{1}\right) \varsubsetneqq \xi^{a}\left(\omega_{2}\right)$ and $\xi^{b}\left(\omega_{2}\right) \varsubsetneqq \xi^{b}\left(\omega_{3}\right)$. 
If $a=b$ then $\xi^{a}\left(\omega_{1}\right) \varsubsetneqq \xi^{a}\left(\omega_{3}\right)$ and $a=\max \left\{i \in\{0, \ldots, k\} \mid \xi^{i}\left(\omega_{1}\right) \neq \xi^{i}\left(\omega_{3}\right)\right\}$ and so $\omega_{1}<_{\mathcal{R}}^{\mathrm{W}} \omega_{3}$. If $a<b$ then $\xi^{b}\left(\omega_{1}\right) \varsubsetneqq \xi^{b}\left(\omega_{3}\right)$ and $b=\max \left\{i \in\{0, \ldots, k\} \mid \xi^{i}\left(\omega_{1}\right) \neq\right.$ $\left.\xi^{i}\left(\omega_{3}\right)\right\}$ and so $\omega_{1}<\mathcal{R} \omega_{3}$. If $a>b$ then $\xi^{i}\left(\omega_{2}\right)=\xi^{i}\left(\omega_{3}\right)$ for all $i \in\{b+1, \ldots, k\}$ and $b+1 \leq a \leq k$; therefore $\xi^{a}\left(\omega_{1}\right) \varsubsetneqq \xi^{a}\left(\omega_{3}\right)$ and $a=\max \left\{i \in\{0, \ldots, k\} \mid \xi^{i}\left(\omega_{1}\right) \neq \xi^{i}\left(\omega_{3}\right)\right\}$ and so $\omega_{1}<{ }_{\mathcal{R}}^{\mathrm{W}} \omega_{3}$.

We now turn to the relationship between the preferred structure $<_{\mathcal{R}}^{\mathrm{w}}$ on worlds and c-representations $\kappa \vec{\eta}$ induced by solutions $\vec{\eta}$ of the restricted $\operatorname{CSP} C R_{o p}(\mathcal{R})$. We first illustrate this relation with our running example.

Example 13 ( $\mathcal{R}_{\text {bird }}$, cont.) For $\kappa \vec{\eta}_{4} \in \operatorname{Sol}\left(C R_{\text {op }}\left(\mathcal{R}_{\text {bird }}\right)\right)$, (cf. Table 2 and Example 8), the relation $<_{\mathcal{R}_{\text {bird }}}^{\mathrm{W}}$ refines the ordering on worlds given by $\kappa \vec{\eta}$, i.e,

$$
\omega<_{\mathcal{R}_{\text {bird }}}^{\mathrm{W}} \omega^{\prime} \text { implies } \kappa \vec{\eta}_{4}(\omega)<\kappa \vec{\eta}_{4}\left(\omega^{\prime}\right)
$$

holds for all worlds over the signature of $\mathcal{R}_{\text {bird }}$.

Due to the special structure of $C R_{o p}(\mathcal{R})$ we can prove the following lemma, stating that the relation pointed out in Example 13 holds for all knowledge bases $\mathcal{R}$ and for all solutions of $C R_{o p}(\mathcal{R})$.

Lemma 4 Let $\mathcal{R}=\left\{\left(B_{i} \mid A_{i}\right) \mid i=1, \ldots, n\right\}$ be a knowledge base with inclusion-maximal partition $\operatorname{OP}(\mathcal{R})=\left(\mathcal{R}_{0}, \ldots, \mathcal{R}_{k}\right)$ and let $\vec{\eta}=\left(\eta_{1}, \ldots, \eta_{n}\right) \in \operatorname{Sol}\left(C R_{o p}(\mathcal{R})\right)$. Then for the c-representation $\kappa \vec{\eta} \in \mathcal{O}(C R(\mathcal{R}))$ given by (6) we have

$$
\omega<<_{\mathcal{R}}^{\mathrm{W}} \omega^{\prime} \text { implies } \kappa \vec{\eta}(\omega)<\kappa_{\vec{\eta}}\left(\omega^{\prime}\right)
$$

for all $\omega, \omega^{\prime} \in \Omega$.

Proof By definition of $<_{\mathcal{R}}^{\mathrm{W}}$, see Definition 12, there exists $m \in\{0, \ldots, k\}$ such that

$$
\begin{aligned}
\xi^{i}(\omega) & =\xi^{i}\left(\omega^{\prime}\right) \quad \forall i \in\{m+1, \ldots, k\}, \\
\xi^{m}(\omega) & \varsubsetneqq \xi^{m}\left(\omega^{\prime}\right) .
\end{aligned}
$$

Then due to (55)

$$
\kappa \vec{\eta}\left(\omega^{\prime}\right)-\kappa \vec{\eta}(\omega)=\sum_{\substack{j \in\{1, \ldots, n\} \\ r_{j} \in \bigcup_{l=0}^{m} \mathcal{R}_{l}, \omega^{\prime} \models A_{j} \overline{B_{j}}}} \eta_{j}-\sum_{\substack{j \in\{1, \ldots, n\} \\ r_{j} \in \bigcup_{l=0}^{m} \mathcal{R}_{l}, \omega \models A_{j} \overline{B_{j}}}} \eta_{j} .
$$

By (56) we can choose $r_{p} \in \mathcal{R}_{m}, p \in\{1, \ldots, n\}$, such that $r_{p} \in \xi^{m}\left(\omega^{\prime}\right)$ but $r_{p} \notin \xi^{m}(\omega)$. Further, since especially $\xi^{m}(\omega) \subseteq \xi^{m}\left(\omega^{\prime}\right)$, we get

$$
\begin{aligned}
& \kappa_{\vec{\eta}}\left(\omega^{\prime}\right)-\kappa_{\vec{\eta}}(\omega) \geq \eta_{p}+\sum_{\substack{j \in\{1, \ldots, n\} \\
r_{j} \in \bigcup^{\prime}=\mathcal{R}_{l}, \omega \mid A_{j} \overline{B_{j}}}} \eta_{j}-\sum_{j \in\{1, \ldots, n\}} \eta_{j} \\
& \geq \eta_{p}-\sum_{\substack{j \in\{1, \ldots, n\} \\
r_{j} \in \bigcup_{l=0}^{m-1} \mathcal{R}_{l}}} \eta_{j}
\end{aligned}
$$

Combining that $\left(\eta_{1}, \ldots, \eta_{n}\right) \in \mathbb{N} \in C R_{o p}(\mathcal{R})$ satisfies (17) with $r_{p} \in \mathcal{R}_{m}$ yields

$$
\eta_{p}>\sum_{\substack{j \in\{1, \ldots, n\} \\ r_{j} \in \bigcup_{l=0}^{m-1} \mathcal{R}_{l}}} \eta_{j}
$$

Therefore $\kappa \vec{\eta}\left(\omega^{\prime}\right)-\kappa \vec{\eta}(\omega)>0$. The claim follows. 
The next technical lemma can be seen as a generalization of a result from [9]. It extends [9, Proposition 15] to the relation $<_{\mathcal{R}}^{\mathrm{W}}$ and to arbitrary knowledge bases, not just knowledge bases only consisting of conditional facts as in [9, Proposition 15]. It tells us that the set of c-representations constructed from $C R_{o p}(\mathcal{R})$ is rich enough to guarantee the existence of a particular c-representation $\kappa \vec{\eta} \in \mathcal{O}(C R(\mathcal{R}))$ fulfilling the constraints as given in the lemma.

Lemma 5 Let $\mathcal{R}=\left\{r_{i}=\left(B_{i} \mid A_{i}\right) \mid i=1, \ldots, n\right\}$ be a consistent knowledge base, let $\omega^{\prime} \in \Omega$ and let $\Omega_{V} \subseteq \Omega$. Assume that $\omega \nless_{\mathcal{R}}^{\mathrm{w}} \omega^{\prime}$ for all $\omega \in \Omega_{V}$. Then there exists $a$ c-representation $\kappa \vec{\eta} \in \mathcal{O}(C R(\mathcal{R}))$ such that

$$
\kappa \vec{\eta}\left(\omega^{\prime}\right) \leq \kappa \vec{\eta}(\omega)
$$

for all $\omega \in \Omega_{V}$.

Proof Throughout this proof we will frequently make use of the following fact which follows from Proposition 5: If $\eta_{i} \in \mathbb{N}, i \in\{1, \ldots, n\}$, satisfy

$$
\eta_{i}>\sum_{\substack{j \in\{1, \ldots, n\} \\ r_{j} \in \bigcup_{l=0}^{m-1} \mathcal{R}_{l}}} \eta_{j}
$$

for all $i \in\{1, \ldots, n\}$ where $m=m(i) \in\{0, \ldots, k\}$ with $r_{i} \in \mathcal{R}_{m}$ then $\left(\eta_{1}, \ldots, \eta_{n}\right)$ is $a$ solution to $C R(\mathcal{R})$. All c-representations constructed in this proof will be constructed from integer impacts satisfying (58). Furthermore let us write $s_{j}:=\left|\mathcal{R}_{j}\right|$ for $j \in\{0, \ldots, k\}$.

Consider $\omega \in \Omega_{V}$ with $\omega \nless_{\mathcal{R}}^{\mathrm{w}} \omega^{\prime}$. Then one of the following cases hold:

(i) $\omega^{\prime}<{ }_{\mathcal{R}}^{\mathrm{W}} \omega$,

(ii) $\xi(\omega)=\xi\left(\omega^{\prime}\right)$,

(iii) $\omega^{\prime} \nless_{\mathcal{R}}^{\mathrm{w}} \omega$ and $\xi(\omega) \neq \xi\left(\omega^{\prime}\right)$.

If (i) holds, then (57) follows from Lemma 4 (since we will use $\eta$ satisfying (58) for the c-representation $\kappa \vec{\eta}$ ) and if (ii) holds, then identity (57) is trivial. Therefore, for $\omega, \omega^{\prime}$ fulfilling (i) or (ii) we have $\kappa \vec{\eta}\left(\omega^{\prime}\right) \leq \kappa \vec{\eta}(\omega)$ for every c-representation $\kappa \vec{\eta}: \Omega \rightarrow \mathbb{N}_{0}$ with $\eta_{i}, i \in\{1,, \ldots, n\}$, satisfying (58).

Therefore, with no loss of generality, let us assume that $\Omega_{V}=\left\{\omega_{1}, \ldots \omega_{l}\right\}$ with $l \in \mathbb{N}$ and for every $\omega \in \Omega_{V}$ there holds

$$
\omega \nless_{\mathcal{R}}^{\mathrm{W}} \omega^{\prime}, \omega^{\prime} \nless_{\mathcal{R}}^{\mathrm{W}} \omega \text { and } \xi(\omega) \neq \xi\left(\omega^{\prime}\right) .
$$

Therefore, applying Lemma 2 twice, once for $\omega_{j} \nless_{\mathcal{R}}^{\mathrm{w}} \omega^{\prime}$ and once for $\omega^{\prime} \nless_{\mathcal{R}}^{\mathrm{W}} \omega_{j}$, yields for every $j \in\{1, \ldots, l\}$ (a unique) $m_{j} \in\{0, \ldots, k\}$ such that

$$
\begin{gathered}
\xi^{i}\left(\omega_{j}\right)=\xi^{i}\left(\omega^{\prime}\right) \quad \forall i \in\left\{m_{j}+1, \ldots, k\right\}, \\
\xi^{m_{j}}\left(\omega_{j}\right) \nsubseteq \xi^{m_{j}}\left(\omega^{\prime}\right) \text { and } \xi^{m_{j}}\left(\omega^{\prime}\right) \nsubseteq \xi^{m_{j}}\left(\omega_{j}\right) .
\end{gathered}
$$

Define the set

$$
I:=\bigcup_{j=1}^{l}\left(\xi^{m_{j}}\left(\omega_{j}\right) \backslash \xi^{m_{j}}\left(\omega^{\prime}\right)\right) .
$$

Due to $\xi^{m_{j}}\left(\omega_{j}\right) \nsubseteq \xi^{m_{j}}\left(\omega^{\prime}\right)$ for every $j \in\{1, \ldots, l\}$ we have that $I$ is not empty. Let us inductively construct the integer impacts $\eta_{i}, i \in\{1, \ldots, n\}$, needed to define a crepresentation $\kappa \in \mathcal{O}(C R(\mathcal{R}))$. Fix $m \in\{0, \ldots, k\}$. In this step our goal is to define $\eta_{i}$ for all $i \in\{1, \ldots, n\}$ with $r_{i} \in \mathcal{R}_{m}$ using that $\eta_{z}$ is already constructed for all $z \in\{1, \ldots, n\}$ such that $r_{z} \in \bigcup_{l=0}^{m-1} \mathcal{R}_{l}$. 
So let us consider any $i \in\{1, \ldots, n\}$ such that $r_{i} \in \mathcal{R}_{m}$. There can occur two cases.

1. If $r_{i} \notin I$, then we define

$$
\eta_{i}:=1+\sum_{\substack{q \in\{1, \ldots, n\} \\ r_{q} \in \bigcup_{l=0}^{m-1} \mathcal{R}_{l}}} \eta_{q}
$$

2. If $r_{i} \in I$ then there exists (a not necessarily unique) $j \in\{1, \ldots, l\}$ such that $m_{j}=m$ and

$$
r_{i} \in \xi^{m_{j}}\left(\omega_{j}\right) \backslash \xi^{m_{j}}\left(\omega^{\prime}\right)
$$

We define

$$
\eta_{i}:=s_{m}\left(1+\sum_{\substack{q \in\{1, \ldots, n\} \\ r_{q} \in \bigcup_{l=0}^{m-1} \mathcal{R}_{l}}} \eta_{q}\right) .
$$

Due to this inductive construction it follows that $\eta_{i} \in \mathbb{N}$ are well defined for $i \in\{1, \ldots, n\}$. (If $m=0$ then $\sum_{\substack{q \in\{1, \ldots, n\} \\ r_{q} \in \bigcup_{l=0}^{m-1} \mathcal{R}_{l}}} \eta_{q}=0$.)

Looking at (58) we obtain that $\kappa \vec{\eta}$ defines a c-representation. It remains to show (57). Fix $j \in \mathbb{N}$. We have to show $\kappa_{\vec{\eta}}\left(\omega_{j}\right)-\kappa_{\vec{\eta}}\left(\omega^{\prime}\right) \geq 0$. There holds (see (61)) that $\omega^{\prime}$ can at most falsify $\left(s_{m_{j}}-1\right)$ conditionals from $\mathcal{R}_{m_{j}}$, i.e.

$$
\left|\left\{q \in\{1, \ldots, n\} ; r_{q} \in \mathcal{R}_{m_{j}}, \omega^{\prime} \models A_{q} \overline{B_{q}}\right\}\right| \leq s_{m_{j}}-1
$$

For every $i \in\{1, \ldots, n\}$ with $r_{i} \in \mathcal{R}_{m_{j}}$ and $\omega^{\prime} \models A_{i} \overline{B_{i}}$ we have $r_{i} \in \xi^{m_{j}}\left(\omega^{\prime}\right)$ and so $r_{i} \notin I$. Therefore $\eta_{i}$ is defined by (63), i.e.

$$
\eta_{i}=1+\sum_{\substack{q \in\{1, \ldots, n\} \\ r_{q} \in \bigcup_{l=0}^{m_{j}-1} \mathcal{R}_{l}}} \eta_{q}
$$

Consequently, combining (66), (67) it follows

$$
\sum_{\substack{q \in\{1, \ldots, n\} \\ r_{q} \in \mathcal{R}_{m_{j}}, \omega^{\prime} \models A_{q} \overline{B_{q}}}} \eta_{q} \leq\left(s_{m_{j}}-1\right)\left(1+\sum_{\substack{q \in\{1, \ldots, n\} \\ r_{q} \in \bigcup_{l=0}^{m_{j}-1} \mathcal{R}_{l}}} \eta_{q}\right) .
$$


Let us choose $r_{p} \in \xi^{m_{j}}\left(\omega_{j}\right) \backslash \xi^{m_{j}}\left(\omega^{\prime}\right)$ where $p \in\{1, \ldots, n\}$. We use (60), that $\eta_{p}$ is defined by (65), and inequality (68) to obtain

$$
\begin{aligned}
& \kappa_{\vec{\eta}}\left(\omega_{j}\right)-\kappa_{\vec{\eta}}\left(\omega^{\prime}\right)=\sum_{\substack{q \in\{1, \ldots, n\} \\
r_{q} \in \bigcup_{l=0}^{m_{j}} \mathcal{R}_{l}, \omega_{j} \models A_{q} \overline{B_{q}}}} \eta_{q}-\sum_{\substack{q \in\{1, \ldots, n\} \\
r_{q} \in \bigcup_{l=0}^{m_{j}} \mathcal{R}_{l}, \omega^{\prime} \models A_{q} \overline{B_{q}}}} \eta_{q} \\
& \geq \eta_{p}-\sum_{\substack{q \in\{1, \ldots, n\} \\
r_{q} \in \mathcal{R}_{m_{j}}, \omega^{\prime} \models A_{q} \overline{B_{q}}}} \eta_{q}-\sum_{\substack{q \in\{1, \ldots, n\} \\
r_{q} \in \bigcup_{l=0}^{m_{j}-1} \mathcal{R}_{l}, \omega^{\prime} \models A_{q} \overline{B_{q}}}} \eta_{q} \\
& \geq s_{m_{j}}\left(1+\sum_{\substack{q \in\{1, \ldots, n\} \\
r_{q} \in \bigcup_{l=0}^{m_{j}-1} \mathcal{R}_{l}}} \eta_{q}\right)-\left(s_{m_{j}}-1\right)\left(1+\sum_{\substack{q \in\{1, \ldots, n\} \\
r_{q} \in \bigcup_{l=0}^{m_{j}-1} \mathcal{R}_{l}}} \eta_{q}\right) \\
& -\sum_{q \in\{1, \ldots, n\}}^{r_{q} \in \bigcup_{l=0} \mathcal{R}_{l}} \eta_{q} \\
& r_{q} \in \bigcup_{l=0}^{m_{j}-1} \mathcal{R}_{l}, \omega^{\prime} \models A_{q} \overline{B_{q}} \\
& =1+\sum_{\substack{q \in\{1, \ldots, n\} \\
r_{q} \in \bigcup_{l=0}^{m_{j}-1} \mathcal{R}_{l}}} \eta_{q}-\sum_{\substack{q \in\{1, \ldots, n\} \\
r_{q} \in \bigcup_{l=0}^{m_{j}-1} \mathcal{R}_{l}, \omega^{\prime} \models A_{q} \overline{B_{q}}}} \eta_{q} \\
& \geq 1 \text {. } \\
& \eta_{q} \\
& q
\end{aligned}
$$

This means $\kappa \vec{\eta}\left(\omega^{\prime}\right) \leq \kappa \vec{\eta}\left(\omega_{j}\right)$ and since $j \in\{1, \ldots, n\}$ was arbitrary, inequality (57) is indeed fulfilled.

In nonmonotonic reasoning from conditional knowledge bases, the notion of conditional indifference is an important concept, stating essentially that worlds falsifying the same conditionals should not be dealt with differently. The following lemma that tells us that worlds falsifying the same sets of conditionals are treated in the same way by $<\mathcal{R}_{\mathcal{R}}^{\mathrm{w}}$.

Lemma 6 (conditional indifference of $<_{\mathcal{R}}^{\mathrm{w}}$ ) Let $\mathcal{R}=\left\{\left(B_{i} \mid A_{i}\right) \mid i=1, \ldots, n\right\}$ be a knowledge base, and let $\omega_{1}, \omega_{2} \in \Omega$ falsify the same sets of conditionals, i.e. $\omega_{1}, \omega_{2} \in \Omega$ satisfy $\xi(\omega)=\xi\left(\omega^{\prime}\right)$. Then $\omega_{1}, \omega_{2}$ behave exactly the same way with respect to $<_{\mathcal{R}}^{\mathrm{w}}$, i.e., for all $\omega \in \Omega$, the following equivalences hold:

$$
\begin{aligned}
& \omega<_{\mathcal{R}}^{\mathrm{w}} \omega_{1} \Longleftrightarrow \omega<_{\mathcal{R}}^{\mathrm{w}} \omega_{2}, \\
& \omega_{1}<_{\mathcal{R}}^{\mathrm{w}} \omega \Longleftrightarrow \omega_{2}<_{\mathcal{R}}^{\mathrm{w}} \omega .
\end{aligned}
$$

Proof The claim follows from $\xi^{i}\left(\omega_{1}\right)=\xi^{i}\left(\omega_{2}\right)$ for all $i \in\{0, \ldots, k\}$.

The technical results established in this section provide useful properties about the preferred structure $<_{\mathcal{R}}^{\mathrm{W}}$ on worlds and its relation to c-representations that will be exploited in the following.

\section{System W}

The preferred structure $<_{\mathcal{R}}^{\mathrm{w}}$ on worlds for a knowledge base $\mathcal{R}$ is defined using both the tolerance information provided by the inclusion-maximal ordered partition $O P(\mathcal{R})$ and information on the set of falsified conditionals. Inference based on $<_{\mathcal{R}}^{\mathrm{w}}$ is called system W inference and is defined as follows. 
Definition 13 (system $\mathrm{W}, \sim \underset{\mathcal{R}}{\mathrm{w}}$ ) Let $\mathcal{R}$ be a knowledge base and let $A, B$ be formulas. Then $B$ is a system $W$ inference from $A$ (in the context of $\mathcal{R}$ ), denoted by

$$
A \sim{ }_{\mathcal{R}}^{\mathrm{w}} B \text { iff } \forall \omega^{\prime} \in \Omega_{A \bar{B}} \exists \omega \in \Omega_{A B} \omega<<_{\mathcal{R}}^{\mathrm{w}} \omega^{\prime} .
$$

A consequence of this definition is that system $\mathrm{W}$ can be represented by the single strict partial order $<_{\mathcal{R}}^{\mathrm{W}}$ on the worlds over the signature of $\mathcal{R}$ which is obtained directly from the ordered partition of $\mathcal{R}$ and the verification/falsification behavior of $\mathcal{R}$.

Example 14 ( $\mathcal{R}_{\text {bird }}$, cont.) Consider again $\mathcal{R}_{\text {bird }}$ from Example 1. Let us show that for $A=b f$ and $B=\bar{p}$ we have $A \sim \stackrel{\mathrm{R}}{\text { bird }}_{\text {bi.e. }}^{\mathrm{w}} B$, that flying birds are usually not penguins. Due to (see Table 8)

$$
\xi(b f \bar{p})=\varnothing, \quad \xi(b f p)=\left\{r_{2}, r_{3}\right\}
$$

it follows that $b f \bar{p}<_{\mathcal{R}_{\text {bird }}}^{\mathrm{W}} b f p$. Therefore, since $\Omega_{A B}=\{b f \bar{p}\}$ and $\Omega_{A \bar{B}}=\{b f p\}$, from (69) it follows that indeed $b f \sim \mathcal{R}_{\text {bird }}^{\mathrm{W}} \bar{p}$.

Note that $b f \sim{ }_{\mathcal{R}}^{c}$ bird $\bar{p}$, i.e., this inference is also a c-inference (cf. Example 3). Therefore, Example 14 presents a c-inference that is also a system $\mathrm{W}$ inference. The following proposition tells us that $A \sim{ }_{\mathcal{R}}^{c} B$ always implies $A \sim{ }_{\mathcal{R}}^{\mathrm{w}} B$.

Proposition 11 (system W captures c-inference) Let $\mathcal{R}$ be a consistent knowledge base. Then we have for all formulas $A, B \in \mathcal{L}$

$$
A \sim{ }_{\mathcal{R}}^{c} B \Longrightarrow A \sim{ }_{\mathcal{R}}^{\mathrm{w}} B
$$

Proof The proof of (70) is by contraposition. So let us assume that we have formulas $A, B \in \mathcal{L}$ with

$$
\exists \omega^{\prime} \in \Omega_{A \bar{B}} \forall \omega \in \Omega_{A B} \omega \nless_{\mathcal{R}}^{\mathrm{w}} \omega^{\prime} .
$$

Our goal is to show $A \not{ }_{\mathcal{R}}^{c} B$. Let us fix $\omega^{\prime} \in \Omega_{A \bar{B}}$ such that (71) holds. Let us define $\Omega_{V}:=$ $\Omega_{A B}$. Then $\omega \nless_{\mathcal{R}}^{\mathrm{w}} \omega^{\prime}$ for all $\omega \in \Omega_{V}$. Due to Lemma 5 there exists a c-representation $\kappa_{\vec{\eta}} \in \mathcal{O}(C R(\mathcal{R}))$ such that $\kappa_{\vec{\eta}}\left(\omega^{\prime}\right) \leq \kappa_{\vec{\eta}}(\omega)$ for all $\omega \in \Omega_{A B}$. This means that $A \not{ }_{\mathcal{R}}{ }^{\vec{\eta}} B$ and so indeed $A \not{ }_{\mathcal{R}}^{c} B$.

The following proposition shows that, in general, system $\mathrm{W}$ strictly extends skeptical c-inference by licensing more entailments.

Proposition 12 (system W goes beyond skeptical c-inference) There exists a knowledge bases $\mathcal{R}$ such that the following holds:

$$
\sim_{\mathcal{R}}^{c} \varsubsetneqq \sim_{\mathcal{R}}^{\mathrm{w}}
$$

Proof Consider the knowledge base $\mathcal{R}^{*}=\{(b \mid a),(b c \mid a)\}$ whose verification/falsification behavior is given by Table 9 . First, due to

$$
\xi(a b \bar{c})=\{(b c \mid a)\} \varsubsetneqq\{(b \mid a),(b c \mid a)\}=\xi(a \bar{b} \bar{c})\}
$$

we obtain $a \bar{c} \sim{ }_{\mathcal{R}^{*}}^{\mathrm{w}} b$.

Making use of the verification/falsification behavior stated in Table 9, we obtain for $C R\left(\mathcal{R}^{*}\right)$

$$
\begin{aligned}
& \eta_{1}>-\eta_{2}, \\
& \eta_{2}>0 .
\end{aligned}
$$


Table 9 Verification/falsification behavior and (generic) c-representation of the knowledge base $\mathcal{R}^{*}$ in the proof of Proposition 12; (v) indicates verification, (f) falsification and (-) non-applicability

\begin{tabular}{lllllllll}
\hline$\omega$ & $a b c$ & $a b \bar{c}$ & $a \bar{b} c$ & $a \bar{b} \bar{c}$ & $\bar{a} b c$ & $\bar{a} b \bar{c}$ & $\bar{a} \bar{b} c$ & $\bar{a} \bar{b} \bar{c}$ \\
\hline$r_{1}=(b \mid a)$ & $\mathrm{v}$ & $\mathrm{v}$ & $\mathrm{f}$ & $\mathrm{f}$ & - & - & - & - \\
$r_{2}=(b c \mid a)$ & $\mathrm{v}$ & $\mathrm{f}$ & $\mathrm{f}$ & $\mathrm{f}$ & - & - & - & - \\
$\kappa \vec{\eta}(\omega)$ & 0 & $\eta_{2}$ & $\eta_{1}+\eta_{2}$ & $\eta_{1}+\eta_{2}$ & 0 & 0 & 0 & 0 \\
\hline
\end{tabular}

Consider the solution vector $\vec{\eta}=\left(\eta_{1}, \eta_{2}\right)=(0,1)$. Then we obtain (see Table 9) for the associated c-representation

$$
\kappa \vec{\eta}(a b \bar{c})=\eta_{2}=\eta_{1}+\eta_{2}=\kappa \vec{\eta}(a \bar{b} \bar{c})
$$

and thus $a \bar{c} \not \nvdash_{\mathcal{R}^{*}}^{c} b$.

Furthermore, every system $\mathrm{Z}$ inference is also a system $\mathrm{W}$ inference.

Proposition 13 (system $\mathrm{W}$ captures system Z) Let $\mathcal{R}$ be a consistent knowledge base. Then we have for all formulas $A, B \in \mathcal{L}$

$$
A \sim \stackrel{\mathcal{R}}{Z}^{Z} B \quad \Longrightarrow A \sim \stackrel{\mathrm{w}}{\mathcal{R}}^{\mathrm{w}} B
$$

Proof Inspecting (5) and (52), we conclude that

$$
\kappa^{Z}(\omega)<\kappa^{Z}\left(\omega^{\prime}\right) \Longrightarrow \omega<<_{\mathcal{R}}^{\mathrm{w}} \omega^{\prime}
$$

holds for arbitrary $\omega, \omega^{\prime} \in \Omega$. Therefore, comparing (4), applied to the ranking function $\kappa^{Z}$, with (69), shows that (73) is fulfilled.

Due to the following proposition we know that there are, in general, strictly more inferences obtained from system $\mathrm{W}$ than from system $\mathrm{Z}$.

Proposition 14 (system W goes beyond system Z) There exists a knowledge bases $\mathcal{R}$ such that the following holds:

$$
\sim{ }_{\mathcal{R}}^{Z} \varsubsetneqq \sim \sim_{\mathcal{R}}^{w}
$$

Proof Consider the knowledge base $\mathcal{R}_{\text {bird }}$ from Example 1. Let us show that for $A=p \bar{b}$ and $B=\bar{f}$ we have $A \sim{ }_{\mathcal{R}_{\text {bird }}}^{\mathrm{w}} B$, i.e., that penguins which are no bird usually do not fly. According to Example 12, we have $p \bar{b} \bar{f}<_{\mathcal{R}_{\text {bird }}}^{\mathrm{w}} p \bar{b} f$. Therefore, since $\Omega_{A B}=\{p \bar{b} \bar{f}\}$

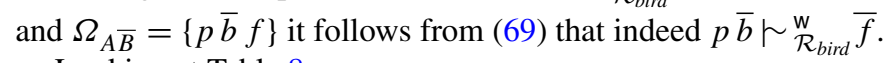

Looking at Table 8 , we see

$$
\kappa^{Z}(A B)=2=\kappa^{Z}(A \bar{B})
$$

and so $p \bar{b} \not \mathcal{R}_{\text {bird }}^{Z} \bar{f}$.

In [23], a preference relation on worlds is defined that is based on structural information by preferring a world $\omega$ to a world $\omega^{\prime}$ if $\omega$ falsifies fewer conditionals than $\omega^{\prime}$ and $\omega^{\prime}$ falsifies at least all conditionals falsified by $\omega$. Using this preference relation, the following entailment relation along the scheme as given by (4) is obtained; we present the definition from [23] in a slightly modified form adapted to our notion $\xi(\omega)$ for the set of conditionals from $\mathcal{R}$ falsified by $\omega$. 
Definition $14\left(\sigma_{\mathcal{R}}\right.$-structural inference [23]) Let $\mathcal{R}=\left\{r_{1}, \ldots, r_{n}\right\}$ with $r_{i}=\left(B_{i} \mid A_{i}\right)$ for $i=1, \ldots, n$ be a knowledge base, $A, B$ formulas, and let $<_{\mathcal{R}}^{\sigma}$ be the relation on worlds given by

$$
\omega<_{\mathcal{R}}^{\sigma} \omega^{\prime} \quad \text { iff } \quad \xi(\omega) \varsubsetneqq \xi\left(\omega^{\prime}\right) .
$$

Then $B$ can be structurally inferred, or $\sigma_{\mathcal{R}}$-inferred, from $A$, written as

$$
A \sim_{\mathcal{R}}^{\sigma} B \text { iff } \forall \omega^{\prime} \in \Omega_{A \bar{B}} \exists \omega \in \Omega_{A B} \omega<_{\mathcal{R}}^{\sigma} \omega^{\prime} .
$$

We can show that every $\sigma_{\mathcal{R}}$-structural inference is also a system $\mathrm{W}$ inference.

Proposition 15 (system $\mathrm{W}$ captures $\sigma_{\mathcal{R}}$-structural inference) Let $\mathcal{R}$ be a consistent knowledge base. Then we have for all formulas $A, B \in \mathcal{L}$

$$
A \sim{ }_{\mathcal{R}}^{\sigma} B \Longrightarrow A \sim{ }_{\mathcal{R}}^{\mathrm{w}} B
$$

Proof Inspecting (52) and (75), we conclude that the implication

$$
\omega<_{\mathcal{R}}^{\sigma} \omega^{\prime} \Longrightarrow \omega<_{\mathcal{R}}^{\mathrm{w}} \omega^{\prime}
$$

holds for arbitrary worlds $\omega, \omega^{\prime} \in \Omega$. Therefore, combining (52) and (76) immediately yields (77).

The consequence of the following proposition is that, in general, there are strictly more entailments obtained from system $\mathrm{W}$ than $\sigma_{\mathcal{R}}$-structural inference.

Proposition 16 (system W goes beyond $\sigma_{\mathcal{R}}$-structural inference) There exists a knowledge bases $\mathcal{R}$ such that the following holds:

$$
\sim_{\mathcal{R}}^{\sigma} \varsubsetneqq \sim \stackrel{\mathrm{w}}{\mathcal{R}}
$$

Proof Consider again the knowledge base $\mathcal{R}_{\text {bird }}$ with the inclusion-maximal partition $O P\left(\mathcal{R}_{\text {bird }}\right)=\left(\mathcal{R}_{0}, \mathcal{R}_{1}\right)($ cf. Example 12) where

$$
\mathcal{R}_{0}=\{(f \mid b)\}, \mathcal{R}_{1}=\{(\bar{f} \mid p),(\bar{f} \mid b p),(b \mid p)\} .
$$

For $\omega=p b f$ we get from Table 8 that $\xi(\omega)=\{(\bar{f} \mid p),(\bar{f} \mid p b)\}$ and

$$
\xi(p b \bar{f})=\{(f \mid b)\}, \quad \xi(p \bar{b} \bar{f})=\{(b \mid p)\} .
$$

Thus, there is no world $\omega^{\prime} \in \Omega$ with $\omega^{\prime} \models p \bar{f}$ and $\omega^{\prime}<_{\mathcal{R}_{\text {bird }}}^{\sigma} \omega$ (which is equivalent to $\left.\xi\left(\omega^{\prime}\right) \varsubsetneqq \xi(\omega)\right)$. Therefore, $p \not \mathcal{R}_{\mathcal{R}_{\text {bird }}}^{\sigma} \bar{f}$.

To show $p \sim{ }_{\mathcal{R}}^{\mathrm{w}} \bar{f}$ fix any $\omega \in \Omega$ with $\omega \models p f$. Then $(\bar{f} \mid p) \in \xi(\omega)$ where $(\bar{f} \mid p) \in R_{1}$. For $\omega^{\prime}=p b \bar{f}$ we have $\omega^{\prime}<_{\mathcal{R}}^{\mathrm{w}} \omega$ due to $\xi\left(\omega^{\prime}\right)=\{(f \mid b)\}$ where $(f \mid b) \in \mathcal{R}_{0}$. Thus indeed $p \sim \mathrm{w}_{\mathcal{R}}^{\mathrm{w}} \bar{f}$.

The following proposition summarizes Propositions 11 - 16. It states that system W strictly extends skeptical c-inference, system $\mathrm{Z}$ inference, and structural inference by licensing, in general, more entailments than each of these three inference modes.

Proposition 17 (system W) For every consistent knowledge base $\mathcal{R}$ we have:

$$
\begin{aligned}
& \sim_{\mathcal{R}}^{c} \subseteq \sim_{\mathcal{R}}^{w} \\
& \sim{ }_{\mathcal{R}}^{Z} \subseteq \sim_{\mathcal{R}}^{w} \\
& \sim{ }_{\mathcal{R}}^{\sigma} \subseteq \sim{ }_{\mathcal{R}}^{w}
\end{aligned}
$$


Furthermore all inclusions in (80) - (82) are strict in the sense that there are knowledge bases $\mathcal{R}_{1}, \mathcal{R}_{2}, \mathcal{R}_{3}$ such that the following holds:

$$
\begin{aligned}
& \sim{ }_{\mathcal{R}_{1}}^{c} \varsubsetneqq \sim \sim_{\mathcal{R}_{1}}^{\mathrm{w}} \\
& \sim{ }^{Z}{ }_{\mathcal{R}_{2}}^{\mathrm{W}} \varsubsetneqq \sim \sim_{\mathcal{R}_{2}}^{\mathrm{w}} \\
& \sim{ }_{\mathcal{R}_{3}}^{\sigma} \varsubsetneqq \sim \sim_{\mathcal{R}_{3}}^{\mathrm{w}}
\end{aligned}
$$

Proof The inclusions in (80) - (82) are shown in Propositions 11, 13, and 15. The strictness of the inclusions in (83) - (85) follows from Propositions 12, 14, and 16.

Because system $\mathrm{W}$ fully captures system $\mathrm{Z}$ and c-inferences, which in turn allows for generally more entailments than system $\mathrm{P}$ inference, we already know that there are system $\mathrm{W}$ entailments that go beyond system P. On the other hand, the next proposition shows that all of the axioms of system $\mathrm{P}$ are satisfied by system $\mathrm{W}$.

Proposition 18 System W inference satisfies system P.

Proof According to Lemma $3,<_{\mathcal{R}}^{\mathrm{w}}$ is a strict transitive relation. Furthermore, since $\Omega$ is finite, the triple $\mathcal{M}^{w}(\mathcal{R})=\left[\Omega, \models,<_{\mathcal{R}}^{\mathrm{w}}\right]$ is a stoppered classical preferential model [31]. Thus, the definition of system $\mathrm{W}$ given by (69) in Definition 13 ensures that system W inference is a preferential inference, hence satisfying system $\mathrm{P}$ (cf. [26, 31]).

After comparing system $\mathrm{W}$ with other established inference methods, let us deal with further of its properties. For this, we express the example scenario sketched in the introduction of this article as a conditional knowledge base.

Example $15\left(\mathcal{R}_{\text {bird }}^{*}\right)$ We extend the alphabet $\Sigma=\{p, b, f\}$ of our running example knowledge base $\mathcal{R}_{\text {bird }}$ from Example 1 with the variable $w$ for having wings and the variable $a$ for being airborne, yielding the alphabet $\Sigma^{*}=\{p, b, f, w, a\}$. We use the knowledge base

$$
\mathcal{R}_{\text {bird }}^{*}=\{(f \mid b),(\bar{f} \mid p),(b \mid p),(w \mid b),(a \mid f)\}
$$

where the conditional $(w \mid b)$ encodes the rule that birds usually have wings, and the conditional $(a \mid f)$ encodes the rule that flying things are usually airborne; the other three conditionals $(f \mid b),(\bar{f} \mid p),(b \mid p)$ are the same as in $\mathcal{R}_{\text {bird }}$.

Note that $\mathcal{R}_{\text {bird }}^{*}$ indeed formalizes the scenario used in Section 1 for sketching the drowning problem, stating that an inference relation suffers from the drowning problem if it does not allow to infer properties of a superclass for a subclass that is exceptional with respect to another property $[11,33]$. The following proposition provides a proof that system $\mathrm{Z}$ suffers from the drowning problem here due to its failure to support the reasonable entailment that penguins usually have wings, because although penguins are exceptional birds with respect to flying, they are not exceptional with respect to having wings.

Proposition 19 System $Z$ inference suffers from the drowning problem in $\mathcal{R}_{\text {bird }}^{*}$ because $p \not \prec \mathcal{R}_{\text {bird }}^{Z} w$.

Proof (i) First, let us show that inclusion-maximal partition $O P\left(\mathcal{R}_{\text {bird }}^{*}\right)=\left(\mathcal{R}_{0}, \mathcal{R}_{1}\right)$ of $\mathcal{R}_{\text {bird }}^{*}$ is given by $\mathcal{R}_{0}=\{(f \mid b),(w \mid b),(a \mid f)\}$ and $\mathcal{R}_{1}=\{(\bar{f} \mid p),(b \mid p)\}$. 
Consider $\omega=\bar{p} b f w a$. It is easy to see that $\omega$ verifies $r_{1}=(f \mid b), r_{4}=(w \mid b), r_{5}=$ $(a \mid f)$ and falsifies no conditional from $\mathcal{R}_{\text {bird }}^{*}$, i.e. that $\left\{r_{1}, r_{4}, r_{5}\right\}$ tolerates $\mathcal{R}_{\text {bird }}^{*}$. Consequently, $\left\{r_{1}, r_{4}, r_{5}\right\} \subseteq \mathcal{R}_{\text {bird }}^{*}$. Moreover, to finish the proof of $\left\{r_{1}, r_{4}, r_{5}\right\}=\mathcal{R}_{\text {bird }}^{*}$ we have to show that for all $\omega$ which verifies either $r_{2}=(\bar{f} \mid p)$ or $r_{3}=(b \mid p)$ we have that $\omega$ falsifies at least one conditional from $\mathcal{R}_{b i r d}^{*}$. The proof is by contradiction.

Assume $\omega$ verifies $(\bar{f} \mid p)$, i.e. $\omega \models p \bar{f}$, but falsifies no conditional from $\mathcal{R}_{\text {bird }}^{*}$. Then $w \models p \bar{f} b$ since $\omega$ does not falsify $(b \mid p)$. However, this is a contradiction to the fact that $\omega$ does not falsify $(f \mid b)$.

Now let us assume that $\omega$ verifies $(b \mid p)$, i.e. $\omega \models p b$, but does not falsify any conditional from $\mathcal{R}_{b i r d}^{*}$. Then $\omega \models p b \bar{f}$ since $\omega$ does not falsify $(\bar{f} \mid p)$. But this is a contradiction to the assumption that $\omega$ does not falsify $(f \mid b)$.

Altogether the proof of $\mathcal{R}_{0}=\{(f \mid b),(w \mid b),(a \mid f)\}$ is complete.

Further, any world $\omega$ with $\omega \models p \bar{f} b$ verifies $r_{2}, r_{3}$ and so indeed $\mathcal{R}_{1}=$ $\{(\bar{f} \mid p),(b \mid p)\}$.

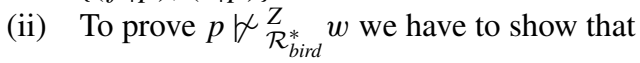

$$
\kappa^{Z}(p w) \geq \kappa^{Z}(p \bar{w}) .
$$

Consider a world $\omega$ with $\omega \models p \bar{f} b \bar{w}$. Then $\omega$ falsifies the conditionals $(f \mid b),(w \mid b)$ from $\mathcal{R}_{0}$ and so $\kappa^{Z}(\omega)=1$ implying $\kappa^{Z}(p \bar{w}) \leq 1$.

On the other hand, consider a world $\omega$ with $\omega \models p w$. We will show that $\kappa^{Z}(\omega) \geq 1$. The proof is by contradiction, so assume that $\kappa^{Z}(\omega)=0$ meaning that $\omega$ falsifies no conditional. It follows $\omega \models p b \bar{f} w$ since $\omega$ falsifies no conditional from $\mathcal{R}_{1}$. However, then $\omega$ falsifies $(f \mid b)$ from $\mathcal{R}_{0}$. Therefore $\kappa^{Z}(\omega) \geq 1$ for all $\omega$ with $\omega \models p w$ implying $\kappa^{Z}(p w) \geq 1$ and so (86) holds. The proof is complete.

In contrast to system $\mathrm{Z}$, the following proposition shows that system $\mathrm{W}$ licenses the inference that penguins usually have wings and thus avoids this drowning phenomenon.

Proposition 20 System $W$ inference does not suffer from the drowning problem in Example 15, i.e., we have $p \sim \underset{\mathcal{R}_{\text {bird }}^{*}}{\mathrm{w}} w$.

Proof We know that the inclusion-maximal partition $O P\left(\mathcal{R}_{\text {bird }}^{*}\right)=\left(\mathcal{R}_{0}, \mathcal{R}_{1}\right)$ of $\mathcal{R}_{\text {bird }}^{*}$ in Example 15 is given by $\mathcal{R}_{0}=\{(f \mid b),(w \mid b),(a \mid f)\}$ and $\mathcal{R}_{1}=\{(\bar{f} \mid p),(b \mid p)\}$.

Consider $\omega \in \Omega$ with $\omega \models p \bar{w}$. Choose an arbitrary $\omega^{\prime} \in \Omega$ with $\omega^{\prime} \models p b \bar{f} w$. We will show $\omega^{\prime}<{ }_{\mathcal{R}_{\text {bird }}^{*}}^{\mathrm{w}} \omega$. Obviously, $\omega^{\prime}$ falsifies only the conditional $(f \mid b)$ which is in $\mathcal{R}_{0}$, written as a formula $\xi(\omega)=\{(f \mid b)\}$. Since $\omega \models p \bar{w}$, we can distinguish the following two cases:

(i) If $\omega \models p \bar{w} f$ then the conditional $(\bar{f} \mid p)$ from $\mathcal{R}_{1}$ is falsified.

(ii) If $\omega \models p \bar{w} \bar{f}$ then we can again distinguish two cases:

(a) If $\omega \models p \bar{w} \bar{f} \bar{b}$ then $(b \mid p)$ from $\mathcal{R}_{1}$ is falsified.

(b) If $\omega \models p \bar{w} \bar{f} b$ then at least $(f \mid b),(w \mid b)$ (both from $\left.\mathcal{R}_{0}\right)$ are falsified.

Due to (52), we thus get $\omega^{\prime}<_{\mathcal{R}_{\text {bird }}^{*}}^{\mathrm{w}} \omega$ in every case, implying $p \sim_{\mathcal{R}_{\text {bird }}^{*}}^{\mathrm{w}} w$.

Thus, avoidance of the drowning problem, distinguishing between inference relations that allow for subclass inheritance only for non-exceptional subclasses (like system Z) and inference relations that allow for subclass inheritance for exceptional subclasses (like cinference) also holds for system $\mathrm{W}$. 
The following observation states that in general, system $\mathrm{W}$ inference cannot be obtained as $\kappa$-entailment induced by a ranking function $\kappa$.

Proposition 21 In general, system $W$ inference cannot be obtained from a ranking function, i.e., there exists a knowledge base $\mathcal{R}$ such that there is no ranking function $\kappa: \Omega \rightarrow \mathbb{N}_{0}^{\infty}$ with $\sim{ }_{\mathcal{R}}^{\mathrm{w}}=\sim^{\kappa}$.

Proof The proof is by contradiction, showing that there is no ranking function $\kappa$ such that $\omega_{1}<{ }_{\mathcal{R}}^{\mathrm{W}} \omega_{2}$ iff $\kappa\left(\omega_{1}\right)<\kappa\left(\omega_{2}\right)$. Assume there is a ranking function $\kappa: \Omega \rightarrow \mathbb{N}_{0}^{\infty}$ with $\omega_{1}<\mathcal{R}_{\mathcal{R}}^{\mathrm{W}} \omega_{2}$ iff $\kappa\left(\omega_{1}\right)<\kappa\left(\omega_{2}\right)$ for $\mathcal{R}_{\text {bird }}$. For $<_{\mathcal{R}}^{\mathrm{w}}$ (cf. Fig. 2) we have $p b f \nless_{\mathcal{R}_{\text {bird }}}^{\mathrm{w}} p \bar{b} f$ and $p \bar{b} f \nless_{\mathcal{R}_{\text {bird }}}^{\mathrm{w}} p b f$ and furthermore $p b f \nless_{\mathcal{R}_{\text {bird }}}^{\mathrm{w}} p \bar{b} \bar{f}$ and $p \bar{b} \bar{f} \kappa_{\mathcal{R}_{\text {bird }}}^{\mathrm{w}} p b f$. Therefore, we obtain $\kappa(p b f)=\kappa(p \bar{b} f)$ and $\kappa(p b f)=\kappa(p \bar{b} \bar{f})$. Thus, $\kappa(p \bar{b} f)=\kappa(p \bar{b} \bar{f})$ which is a contradiction to $p \bar{b} \bar{f}<{ }_{\mathcal{R}_{\text {bird }}}^{\mathrm{w}} p \bar{b} f$.

An important chracteristic of system $\mathrm{Z}$ is given by its property of relying on the unique minimal ranking model $\kappa_{\mathcal{R}}^{Z}$ accepting a knowledge base $\mathcal{R}$. Moreover, if $m$ is the maximal value of $\kappa_{\mathcal{R}}^{Z}(\omega)$ for all worlds $\omega$, then for every $m^{\prime} \in \mathbb{N}$ with $m^{\prime} \leq m$ there is a world $\omega^{\prime}$ such that $\kappa_{\mathcal{R}}^{Z}\left(\omega^{\prime}\right)=m^{\prime}$. For ranking functions with this property of not having any empty layers, there is a bijective correspondence to total preorders on the worlds over the signature of $\mathcal{R}$, given by $\omega^{\prime} \preceq \omega$ iff $\kappa_{\mathcal{R}}^{Z}\left(\omega^{\prime}\right) \leq \kappa_{\mathcal{R}}^{Z}(\omega)$. Thus, the system $\mathrm{Z}$ ranking function can be represented equivalently by single total preorder. Although Proposition 21 states that in general, there is no OCF for representing system $\mathrm{W}$ inference, in contrast to c-inference that requires to take sets of ranking models into account, system $\mathrm{W}$ still has the simple representation by the single strict partial order $<_{\mathcal{R}}^{\mathrm{w}}$ of the worlds over the signature of $\mathcal{R}$.

\section{Conclusions and future work}

Skeptical c-inference is an inductive inference method for completing the explicit knowledge given by a conditional knowledge base. We presented a criterion on a knowledge base $\mathcal{R}$ such that using $|\mathcal{R}|-1$ as an upper bound is sufficient for realizing c-inference for $\mathcal{R}$ by a finite domain constraint system. Given any verification/falsification behavior of conditionals on worlds, we developed a constructive approach yielding a knowledge base realizing this behavior. In contrast to the previous conjecture that a maximal impact $u=|\mathcal{R}|$ is sufficient for $\mathcal{R}$, due to our results presented here, we know that there is no polynomial bound for $u \in \mathbb{N}$ to be minimally sufficient for all knowledge bases with $n$ conditionals for realizing skeptical c-inference over $\mathcal{R}$. Inspired by our findings, we introduced the preferred structure relation on worlds as the core ingredient of a new inference relation, called system $\mathrm{W}$ inference. In contrast to skeptical c-inference which requires to consider all solutions of a complex constraint satisfaction problem, system $\mathrm{W}$ can be represented by a single strict partial order on the worlds. We proved that system $\mathrm{W}$ inference fully captures and strictly extends both skeptical c-inference and also system $\mathrm{Z}$ inference. Furthermore, system $\mathrm{W}$ exhibits many other properties desirable for nonmonotonic reasoning, like satisfying the axioms of system $\mathrm{P}$ or avoiding the drowning problem.

We are currently working on investigating system $\mathrm{W}$ with respect to further properties put forward for nonmonotonic reasoning, e.g., regarding syntax splitting [21, 22, 32, 34]. We are also empirically evaluting system $\mathrm{W}$ with the reasoning platform InfOCF $[6,28]$ and investigating to what extent compilation techniques developed for implementing c-inference [10] can be employed for system W. The problem of establishing an upper bound for knowledge 
bases that is sufficient to realize skeptical c-inference remains open and will be addressed in future work.

Funding Open Access funding enabled and organized by Projekt DEAL.

Open Access This article is licensed under a Creative Commons Attribution 4.0 International License, which permits use, sharing, adaptation, distribution and reproduction in any medium or format, as long as you give appropriate credit to the original author(s) and the source, provide a link to the Creative Commons licence, and indicate if changes were made. The images or other third party material in this article are included in the article's Creative Commons licence, unless indicated otherwise in a credit line to the material. If material is not included in the article's Creative Commons licence and your intended use is not permitted by statutory regulation or exceeds the permitted use, you will need to obtain permission directly from the copyright holder. To view a copy of this licence, visit http://creativecommons.org/licenses/by/4.0/.

\section{References}

1. Adams, E.W.: The logic of conditionals: an application of probability to deductive logic. Synthese library. Springer Science+Business media, dordrecht NL (1975)

2. Beierle, C., Eichhorn, C., Kern-Isberner, G.: Skeptical inference based on c-representations and its characterization as a constraint satisfaction problem. In: FoIKS-2016, volume 9616 of LNCS, pages 65-82. Springer (2016)

3. Beierle, C., Eichhorn, C., Kern-Isberner, G., Kutsch, S.: Skeptical, weakly skeptical, and credulous inference based on preferred ranking functions. In: Kaminka, G.A., Fox, M., Bouquet, P., Hüllermeier, E., Dignum, V., Dignum, F., van Harmelen, F. (eds.) Proceedings 22nd European Conference on Artificial Intelligence, ECAI-2016, volume 285 of Frontiers in Artificial Intelligence and Applications, pages 1149-1157. IOS Press (2016)

4. Beierle, C., Eichhorn, C., Kern-Isberner, G., Kutsch, S.: Properties of skeptical c-inference for conditional knowledge bases and its realization as a constraint satisfaction problem. Ann. Math. Artif. Intell. 83(3-4), 247-275 (2018)

5. Beierle, C., Eichhorn, C., Kern-Isberner, G., Kutsch, S.: Properties and interrelationships of skeptical, weakly skeptical, and credulous inference induced by classes of minimal models. Artif. Intell., 297 (2021)

6. Beierle, C., Eichhorn, C., Kutsch, S.: A practical comparison of qualitative inferences with preferred ranking models. KI - Künstliche Intelligenz 31(1), 41-52 (2017)

7. Beierle, C., Kern-Isberner, G.: Semantical investigations into nonmonotonic and probabilistic logics. Ann. Math. Artif. Intell. 65(2-3), 123-158 (2012)

8. Beierle, C., Kutsch, S.: Regular and sufficient bounds of finite domain constraints for skeptical cinference. In: Benferhat, S., Tabia, K., Ali, M. (eds.) Advances in Artificial Intelligence: From Theory to Practice, volume 10350 of LNCS, pages 477-487. Springer (2017)

9. Beierle, C., Kutsch, S.: Computation and comparison of nonmonotonic skeptical inference relations induced by sets of ranking models for the realization of intelligent agents. Appl. Intell. 49(1), 28-43 (2019)

10. Beierle, C., Kutsch, S., Sauerwald, K.: Compilation of static and evolving conditional knowledge bases for computing induced nonmonotonic inference relations. Ann. Math. Artif. Intell. 87(1-2), 5-41 (2019)

11. Benferhat, S., Cayrol, C., Dubois, D., Lang, J., Prade, H.: Inconsistency Management and Prioritized Syntax-Based Entailment. Inproceedings of the Thirteenth International Joint Conference on Artificial Intelligence (IJCAI'93), Volume 1, Pages 640-647, San Francisco, CA, USA. Morgan Kaufmann Publishers (1993)

12. Benferhat, S., Dubois, D., Prade, H.: Representing default rules in possibilistic logic. Inproceedings 3th International Conference on Principles of Knowledge Representation and Reasoning KR'92, pp 673-684 (1992)

13. de Finetti, B.: La prévision, ses lois logiques et ses sources subjectives. Ann. Inst. H. Poincaré 7(1), 1-68 (1937). English translation in Studies in Subjective Probability, ed. H. Kyburg and H.E. Smokler, 1974, 93-158. New York: Wiley \& Sons

14. Dubois, D., Prade, H.: Conditional objects as nonmonotonic consequence relationships. Special Issue on Conditional Event Algebra. IEEE Transactions on Systems Man and Cybernetics 24(12), 1724-1740 (1994) 
15. Dubois, D., Prade, H.: Possibility theory and its applications: Where do we stand?. In: Kacprzyk, J., Pedrycz, W. (eds.) Springer Handbook of Computational Intelligence, pages 31-60. Springer, Berlin (2015)

16. Goldszmidt, M., Pearl, J.: On the consistency of defeasible databases. Artif. Intell. 52(2), 121-149 (1991)

17. Goldszmidt, M., Pearl, J.: Qualitative probabilities for default reasoning, belief revision, and causal modeling. Artif. Intell. 84(1-2), 57-112 (1996)

18. Hawthorne, J., Makinson, D.: The quantitative/qualitative watershed for rules of uncertain inference. Stud. Logica. 86(2), 247-297 (2007)

19. Kern-Isberner, G.: Conditionals in nonmonotonic reasoning and belief revision, volume 2087 of LNAI Springer (2001)

20. Kern-Isberner, G.: A thorough axiomatization of a principle of conditional preservation in belief revision. Ann. Math. Artif. Intell. 40(1-2), 127-164 (2004)

21. Kern-Isberner, G., Beierle, C., Brewka, G.: Syntax splitting = relevance + independence: New postulates for nonmonotonic reasoning from conditional belief bases. In: KR-2020, pp 560-571 (2020)

22. Kern-Isberner, G., Brewka, G.: Strong syntax splitting for iterated belief revision. Inproceedings of the Twenty-Sixth International Joint Conference on Artificial Intelligence, IJCAI 2017, Melbourne, Australia, August 19-25, 2017, pp 1131-1137 (2017)

23. Kern-Isberner, G., Logica, C.: Eichhorn. Structural inference from conditional knowledge bases. Studia Special Issue Logic and Probability:, Reasoning in Uncertain Environments 102(4), 751-769 (2014)

24. Komo, C., Beierle, C.: Nonmonotonic inferences with qualitative conditionals based on preferred structures on worlds. In: Schmid, U., Klügl, F., Wolter, D. (eds.) KI, Advances in Artificial Intelligence - 43rd German Conference on AI, Bamberg, Germany, September 21-25, 2020, Proceedings, volume 12325 of LNCS, pages 102-115, Springer, 2020 (2020)

25. Komo, C., Beierle, C.: Upper and lower bounds for finite domain constraints to realize skeptical cinference over conditional knowledge bases. In: International Symposium on Artificial Intelligence and Mathematics (ISAIM), Fort lauderdale, FL, USA, January 6-8, 2020 (2020)

26. Kraus, S., Lehmann, D., Magidor, M.: Nonmonotonic reasoning, preferential models and cumulative logics. Artif. Intell. 44, 167-207 (1990)

27. Kutsch, S., Beierle, C.: InfOCF-Web: An online tool for nonmonotonic reasoning with conditionals and ranking functions. In: Zhou, Z. (ed.) Proceedings of the Thirtieth International Joint Conference on Artificial Intelligence, IJCAI 2021, Virtual Event / Montreal, Canada, 19-27 August 2021, pages 4996-4999. ijcai.org (2021)

28. Kutsch, S., Beierle, C.: Semantic classification of qualitative conditionals and calculating closures of nonmonotonic inference relations. Int. J. Approx. Reason. 130, 297-313 (2021)

29. Lehmann, D., Magidor, M.: What does a conditional knowledge base entail Artif. Intell. 55, 1-60 (1992)

30. Lewis, D.: Counterfactuals. Harvard University Press, Cambridge (1973). Mass.

31. Makinson, D.: General patterns in nonmonotonic reasoning. In: Gabbay, D., Hogger, C., Robinson, J. (eds.) Handbook of Logic in Artificial Intelligence and Logic Programming, volume 3, pages 35-110. Oxford University Press (1994)

32. Parikh, R.: Beliefs, belief revision, and splitting languages. Logic, Language, and Computation 2, 266278 (1999)

33. Pearl, J.: System Z: A natural ordering of defaults with tractable applications to nonmonotonic reasoning. In: Parikh, R. (ed.) Proceedings of the 3rd conference on Theoretical aspects of reasoning about knowledge (TARK1990), pages 121-135, San Francisco, CA, USA. Morgan Kaufmann Publishers Inc (1990)

34. Peppas, P., Williams, M.-A., Chopra, S., Foo, N.Y.: Relevance in belief revision. Artif. Intell. 229(1-2), 126-138 (2015)

35. Spohn, W.: Ordinal Conditional Functions: A Dynamic Theory of Epistemic States. In: Causation in Decision, Belief Change and Statistics: Proceedings of the Irvine Conference on Probability and Causation, volume 42 of The Western Ontario Series in Philosophy of Science, pages 105-134, Dordrecht, NL. Springer Science+Business Media (1988)

36. Spohn, W.: The laws of belief: ranking theory and its philosophical applications. Oxford univ. press, Oxford UK (2012)

37. Thorn, P.D., Eichhorn, C., Kern-Isberner, G., Schurz, G.: Qualitative Probabilistic Inference with Default Inheritance for Exceptional Subclasses. In: Beierle, C., Kern-Isberner, G., Ragni, M., Stolzenburg, F. (eds.) Proceedings of the 5th Workshop on Dynamics of Knowledge and Belief (DKB-2015) and the 4th Workshop KI \& Kognition (KIK-2015) co-located with 38th German Conference on Artificial Intelligence (KI-2015), volume 1444 of CEUR Workshop Proceedings (2015)

Publisher's note Springer Nature remains neutral with regard to jurisdictional claims in published maps and institutional affiliations. 